\title{
Multiple Schramm-Loewner Evolutions and Statistical Mechanics Martingales
}

\author{
Michel Bauer $^{a}$, Denis Bernard ${ }^{a}{ }^{1}$, Kalle Kytölä ${ }^{b}$ \\ michel.bauer@cea.fr; denis.bernard@cea.fr; kalle.kytola@helsinki.fi \\ a Service de Physique Théorique de Saclay \\ CEA/DSM/SPhT, Unité de recherche associée au CNRS \\ CEA-Saclay, 91191 Gif-sur-Yvette, France. \\ ${ }^{b}$ Department of Mathematics, P.O. Box 68 \\ FIN-00014 University of Helsinki, Finland.
}

\begin{abstract}
A statistical mechanics argument relating partition functions to martingales is used to get a condition under which random geometric processes can describe interfaces in $2 \mathrm{~d}$ statistical mechanics at criticality. Requiring multiple SLEs to satisfy this condition leads to some natural processes, which we study in this note. We give examples of such multiple SLEs and discuss how a choice of conformal block is related to geometric configuration of the interfaces and what is the physical meaning of mixed conformal blocks. We illustrate the general ideas on concrete computations, with applications to percolation and the Ising model.
\end{abstract}

\footnotetext{
${ }^{1}$ Member of C.N.R.S
} 


\section{Contents}

\begin{tabular}{lll}
\hline & Introduction & 3
\end{tabular}

2 Basics of Schramm-Löwner evolutions: Chordal SLE 6

\begin{tabular}{|lll}
\hline A proposal for multiple SLEs & 7
\end{tabular}

3.1 The basic equations . . . . . . . . . . . . 7

3.2 Arch probabilities . . . . . . . . . . . . . . . 8

$\begin{array}{lll}4 \text { First comments } & 10\end{array}$

4.1 Statistical mechanics interpretation . . . . . . . . . . . . 10

4.2 SLE as a special case of 2SLE . . . . . . . . . . . . . 10

4.3 Making sense . . . . . . . . . . . . . . . . . 12

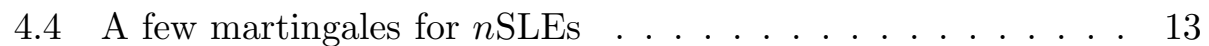

4.5 Classical limit . . . . . . . . . . . . . . . . . . . . . . 14

4.6 Relations with other work . . . . . . . . . . . . 15

$\begin{array}{lll}5 & \text { CFT background } & 16\end{array}$

6 Martingales from statistical mechanics 19

6.1 Tautological martingales . . . . . . . . . . . . . . . . . 19

6.2 Simplifving tautological martingales . . . . . . . . . . . 21

7 Derivation of the proposal 22

7.1 The three ingredients . . . . . . . . . . . . . . . . . 23

7.2 Computation of the Ito derivative of $\left\langle\prod^{l}{ }_{-1} \varphi_{\delta}\left(Y^{(\alpha)}\right)\right\rangle_{+} \ldots .23$

7.3 Main claim . . . . . . . . . . . . . . . . . . 25

7.4 The moduli space . . . . . . . . . . . . . . . . . 26

8 Multiple SLEs describing several interfaces 28

8.1 Double SLEs . . . . . . . . . . . . . . . . . 28

8.1.1 2SLEs and Bessel processes . . . . . . . . . . 28

8.1 .2 A mixed case for $2 \mathrm{SLE} \ldots \ldots \ldots$. . . . . . . . 30

8.2 Triple and/or quadruple SLEs . . . . . . . . . . . . . . . 31

8.2.1 3SLE (pure) configurations . . . . . . . . . 31

8.2 .2 Arch probabilities . . . . . . . . . . . . 33

8.3 Applications to percolation and Ising model . . . . . . . . . . 35

8.4 nSLEs and bevond . . . . . . . . . . . . . . 36 


\section{Introduction}

Growth phenomena are ubiquitous around us. They have both very practical applications and theoretical relevance. But they are rarely easy to study analytically and very few rigorous or exact results are known. In two dimensions, the description of a growing domain is often obtained indirectly through the description of a family of univalent holomorphic representations, leading quite generally to equations known under the name of Loewner chains. These techniques, based on the Riemann mapping theorem, are conceptually important but usually far from making the problem tractable.

In the last few years, Loewner chains have been discovered which have a large hidden symmetry - conformal invariance - that makes them more amenable to an exact treatment [1]. These are known under the name of Stochastic or (Schramm) Loewner evolutions SLE.

Their mathematical elegance and simplicity is not their sole virtue. They are also natural candidates to describe the continuum limit of an interface in two-dimensional statistical mechanics models at criticality. At the critical temperature and in the continuum limit, the system is believed to be conformally invariant and physicists have developed many powerful techniques, known under the name conformal field theory or CFT, to deal with local questions in a conformally invariant $2 \mathrm{~d}$ system. However, nonlocal objects like interfaces posed new nontrivial problems that finally SLE could attack in a systematic way [5, 4]. The connection between CFT and SLE is now well understood [8, 7, 9, 11, 12, 14, and the interplay between the two approaches has proved fruitful.

The way SLE describes an interface deserves some comments. As a guiding example, consider the Ising model in a simply connected domain, say on the hexagonal lattice. Suppose that the boundary is split in two arcs with endpoints say $a$ and $b$ and impose that on one arc the spins are up and on the other one the spins are down. In this situation each sample exhibits an interface. It joins the two points where the boundary conditions change and splits the domain in two pieces, one with all spins up on its boundary and one with all spins down. This interface fluctuates from sample to sample. What SLE teaches us is the following. Instead of describing the interface between $a$ and $b$ at once, SLE views it as a curve starting from say $a$ and growing toward $b$. And SLE describes the distribution for the addition of an infinitesimal piece of interface when the beginning of the interface is already known. So the description is in terms of a growth process even if there was no growth process to start with. 
As mentioned above, the probabilistic aspects of SLE as well as its connections with conformal field theory are now fairly well understood. However, some fundamental questions remain, again directly related to natural questions in the statistical mechanics framework.

The one we shall concentrate on in this note is what happens when, due to boundary conditions, the system contains several interfaces. Proposals for multiple SLEs have already been made in the literature [15, 16, but our results point to a different picture. The simplest situation is in fact when there is only one interface but we want to deal with its two ends symmetrically so that two growth processes will interact with each other. Remember that standard SLE deals with the two ends of the interface asymmetrically. This has a price : time reversed SLE is an intricate object.

As a guiding example for more than one interface, consider again the Ising model in a simply connected domain on the hexagonal lattice. If one changes boundary conditions from up to down to up and so on $n=2 m$ times along the boundary, each sample will exhibit $m$ interfaces, starting and ending on the boundary at points where the boundary conditions change, forming a so-called arch system. However, the interfaces will fluctuate from sample to sample and so does even the topology of the arch system. This topology, for instance, is an observable that is trivial for a single SLE.

Our description will again be in terms of growth processes and Loewner chains. For standard SLE, the driving parameter is a continuous martingale and the tip of the curve separates two different states of the system (up and down spins for Ising), leading to a well defined boundary changing operator in statistical mechanics. The relation between the stochastic Loewner equation and the boundary changing operator comes via a diffusion equation that they share in common.

For multiple SLEs, we expect that for short time scales each curve grows under the influence of an independent martingale. At its tip stands the same boundary changing operator. But we also expect drift terms, describing interactions between the curves.

The possibility of different arch topologies makes it even more natural to have a description with one curve growing at each boundary changing point so that each of them is on the same footing. So $m$ interfaces are described by $n=2 m$ growth processes of "half-interfaces" that finally pair in a consistent way to build arches.

In statistical mechanics, each arch system has a well defined probability to show up. The law governing this finite probability space is again described by a Boltzmann weight which is nothing but a partial partition function.

Our starting point is the reconsideration of the role of Boltzmann weights 
and partition functions in statistical mechanics and their simple but crucial relationship with probabilistic martingales. This allows us to ask the question "by what kind of stochastic differential equations can one describe multiple SLEs ?" by imposing a martingale property and conformal invariance. This puts strong constraints on the drift terms and our main result is a description of the family of drift terms that are compatible with the basic rules of statistical mechanics. Each drift is expressed in terms of the partition function of the system. This partition function is given by a sum of Boltzmann weights for configurations that satisfy certain boundary conditions : at the starting points of the curves the boundary conditions change. The partition function depends on the position of these changes, so up to normalization, the partition function is in fact a correlation function. It satisfies a number of partial differential equations (one equation for each point) that are related to the diffusion equations for the multi SLE process. The solutions form a finite dimensional vector space. The positivity constraint satisfied by physical partition functions singles out a cone which is expected, again guided by statistical mechanics, to have the same dimension of the underlying vector space and to be the convex hull of a family of half lines, so that a generic hyperplane section of the cone is a simplex.

So geometrically, the drift terms are parametrized by a cone. Extremal drifts, i.e. drifts corresponding to extremal lines in this cone, lead to processes for which the final pattern formed by the growing curves is a given arch system. Drifts inside the simplex give rise to stochastic processes where the asymptotic arch system fluctuates from sample to sample. A crucial role to construct martingales describing interesting events is played by the short distance expansion in conformal field theory because this is what tells which terms in the partition function become dominant when an arch closes, i.e. when two driving processes of the multi SLE hit each other.

The vector space of solution of the differential equations for the partition functions has a famous basis indexed by Dyck paths, which are in one to one correspondence with arch systems. But the basis elements do not in general correspond to extremal partition functions. We shall give a rationale for computing the matrix elements for the change of basis and compute a number of them, but we have no closed general formula.

We shall illustrate our proposal with concrete computations for 1 to 3 interfaces with applications to percolation and the Ising model. We shall also discuss the classical (deterministic) limit $\kappa \rightarrow 0^{+}$, where only extremal drifts survive.

The notes also cover the case when a number of boundary changes are very close to each other but the system is conditioned so that they do not 
pair with each other. The details are in the main text.

Our description is rather flexible in the sense that the speed of growth of each piece of interface can be tuned. Certain limiting cases lead to previously known processes which are examples of $\operatorname{SLE}(\kappa, \rho)$ processes.

It is appropriate here to stress that many of the probabilistic properties of the solutions of the stochastic differential equations that we introduce are conjectural at this point. We have made some consistency checks ${ }^{2}$ and the whole pattern is elegant, but our confidence comes more from our familiarity with conformal field theory and statistical mechanics.

\section{Basics of Schramm-Löwner evolutions: Chordal SLE}

Let us briefly recall what is meant by the chordal SLE - detailed studies can be found in 2] or 3]. The chordal SLE process in the upper half plane $\mathbb{H}$ is defined by the ordinary differential equation

$$
\frac{\mathrm{d}}{\mathrm{d} t} g_{t}(z)=\frac{2}{g_{t}(z)-\xi_{t}}
$$

where the initial condition is $g_{0}(z)=z \in \mathbb{H}$ and $\xi_{t}=\sqrt{\kappa} B_{t}$ is a Brownian motion with variance parameter $\kappa \geq 0$. Let $\tau_{z} \leq \infty$ denote the explosion time of (11) with initial condition $z$ and define the hull at time $t$ by $K_{t}:=$ $\overline{\left\{z \in \mathbb{H} \mid \tau_{z}<t\right\}}$. Then $\left(K_{t}\right)_{t \geq 0}$ is a family of growing hulls, $K_{s} \subset K_{t}$ for $s<t$. The complement $\mathbb{H} \backslash K_{t}$ is simply connected and $g_{t}$ is the unique conformal mapping $\mathbb{H} \backslash K_{t} \rightarrow \mathbb{H}$ with $g_{t}(z)=z+o(1)$ at $z \rightarrow \infty$. One defines the SLE trace by $\gamma_{t}=\lim _{\epsilon \downarrow 0} g_{t}^{-1}\left(\xi_{t}+i \epsilon\right)$. The trace is a continuous path in $\overline{\mathbb{H}}$ and it generates the hulls in the sense that $\mathbb{H} \backslash K_{t}$ is the unbounded component of $\mathbb{H} \backslash \gamma_{[0, t]}$. For $\kappa \leq 4$ the trace is a non-self-intersecting path and it doesn't hit $\partial \mathbb{H}=\mathbb{R}$ for $t>0$ so $K_{t}=\gamma_{[0, t]}$. For $4<\kappa<8$ a typical point $z \in \mathbb{H}$ is swallowed, i.e. $z \in K_{t}$ for large $t$ but $z \notin \gamma_{[0, \infty)}$. In the parameter range $\kappa \geq 8$ the trace is space filling, $\gamma_{[0, \infty)}=\overline{\mathbb{H}}$. Let us point out that no statistical mechanics models seem to correspond to $\kappa>8$.

In the definition of chordal SLE we took the usual parametrization of time. From equation (11) we see that $g_{t}(z)=z+2 t z^{-1}+\mathcal{O}\left(z^{-2}\right)$, which means (this could be taken as a definition) that the capacity of $K_{t}$ from

\footnotetext{
${ }^{2}$ For instance, Dubédat has derived general "commutation criteria" 16 for multiple SLEs. The processes we study are a special class satisfying commutation. This class extends vastly the special solution found by Dubédat, which in our language corresponds to self avoiding SLEs moving to infinity.
} 
infinity is $2 t$. Since the capacity goes to infinity as $t \rightarrow \infty$, the hulls $K_{t}$ are not contained in any bounded subset of $\overline{\mathbb{H}}$.

If the parametrization of time is left arbitrary, Schramm's argument yields :

$$
\mathrm{d} g_{s}(z)=\frac{2 \mathrm{~d} q_{s}}{g_{s}(z)-M_{s}},
$$

where $M_{s}$ is a continuous martingale with quadratic variation $\kappa q_{s}$ (an increasing function going to infinity with $s$ ). In this formula, both $q_{s}$ and $M_{s}$ are random objects. The capacity of $K_{s}$ is $2 q_{s}$. But this is not really more general than eq.(11) which is recovered by a random time change.

\section{A proposal for multiple SLEs}

The motivations for our proposal require a good amount of background, but the proposal and its main features themselves can be easily stated. We gather them in this section. Some of the results are conjectures. The rest of the paper will then be split into sections whose purpose will be either to motivate our proposal in general, or to prove its correctness in certain special but nontrivial cases by explicit computations.

\subsection{The basic equations}

We propose to describe the local growth of $n$ interfaces in CFT, labeled by an integer $i=1, \cdots, n$ and joining fixed points on the boundary by a Loewner chain. We assume that $0 \leq \kappa<8$ in the following. We list the set of necessary conditions and equations.

Conformal invariance: The measure on $n$ SLE is conformally invariant. Hence it is enough to give its definition when the domain $D$ is the upper half plane $\mathbb{H}$ in the hydrodynamical normalization.

Universe: The basic probabilistic objects are $n$ (continuous, local) martingales $M_{t}^{(i)}, i=1, \cdots, n$ with quadratic variation $\kappa q_{t}^{(i)}$ absolutely continuous with respect to $\mathrm{d} t$ and vanishing cross variation, defined on an appropriate probability space. By a time change we can and shall assume that $\sum_{i} q_{t}^{(i)} \equiv t$

Driving processes: The processes $X_{t}^{(i)}$ are solutions of the stochastic dif- 
ferential equations

$$
\mathrm{d} X_{t}^{(i)}=\mathrm{d} M_{t}^{(i)}+\kappa \mathrm{d} q_{t}^{(i)}\left(\partial_{x_{i}} \log Z\right)\left(X_{t}^{(1)}, \cdots, X_{t}^{(n)}\right)+\sum_{j \neq i} \frac{2 \mathrm{~d} q_{t}^{(j)}}{X_{t}^{(i)}-X_{t}^{(j)}} .
$$

The initial conditions are $X_{0}^{(i)}=X_{i}$ ordered in such a way that $X_{1}<X_{2}<$ $\cdots<X_{n}$.

Loewner chain: The map $f_{t}$ uniformizing the complement of the hulls satisfies

$$
\mathrm{d} f_{t}(z)=\sum_{i} \frac{2 \mathrm{~d} q_{t}^{(i)}}{f_{t}(z)-X_{t}^{(i)}}
$$

The initial condition is $f_{0}(z)=z$. With our conventions, the total capacity of the growing hulls at time $t$ is $2 t$.

Auxiliary function: The system depends on a function $Z\left(x_{1}, \cdots, x_{n}\right)$ which has to fulfill the following requirements :

i) $Z\left(x_{1}, \cdots, x_{n}\right)$ is defined and positive for $x_{1}<x_{2}<\cdots<x_{n}$,

ii) $Z\left(x_{1}, \cdots, x_{n}\right)$ is translation invariant and homogeneous. Its weight is $h_{n-2 m}(\kappa)-n h_{1}(\kappa)$ for some nonnegative integer $m \leq n / 2$, where ${ }^{3}$

$$
2 \kappa h_{m}(\kappa) \equiv m(2(m+2)-\kappa) .
$$

iii) $Z\left(x_{1}, \cdots, x_{n}\right)$ is annihilated by the $n$ differential operators

$$
\mathcal{D}_{i}=\frac{\kappa}{2} \partial_{x_{i}}^{2}+2 \sum_{j \neq i}\left[\frac{1}{x_{j}-x_{i}} \partial_{x_{j}}-\frac{h_{1}(\kappa)}{\left(x_{j}-x_{i}\right)^{2}}\right] .
$$

We call this system of equations the $n$ SLE system for $n$ curves joining together the points $X_{1}, \cdots, X_{n}$ and possibly the point at infinity. Systems for radial and dipolar versions of $n$ SLE could be defined analogously.

\subsection{Arch probabilities}

It is known from CFT that (relaxing the positivity constraint), the solutions to $i)$, $i i)$, iii) form a vector space of dimension $d_{n, m} \equiv\left(\begin{array}{c}n \\ m\end{array}\right)-\left(\begin{array}{c}n \\ m-1\end{array}\right)=$ $(n+1-2 m) \frac{n !}{m !(n-m+1) !}$.

The positive solutions form a cone and from the statistical mechanics interpretation, we conjecture that this cone has the same dimension and is

\footnotetext{
${ }^{3}$ A more traditional notation for $h_{m}(\kappa)$ is $h_{1, m+1}$ in the physics literature.
} 
generated by (i.e. is the convex hull of) $d_{n, m}$ half lines (extremal lines, pure states in the sense of statistical mechanics) so that a transverse section of the cone is a simplex. So each solution $Z$ can be written in a unique way as a sum of extremal states.

The numbers $d_{n, m}$ have many many combinatorial interpretations, but the one relevant for us is the following. Draw $n+1$ points $X_{1}<X_{2} \cdots<$ $X_{n}<\infty$ ordered cyclically on the (extended) real line bounding the upper half plane $\mathbb{H}$. Consider $n-m$ disjoint curves in $\mathbb{H}$ such that each $X_{i}$ is an end point of exactly 1 curve and $\infty$ is an end point of exactly $n-2 m$ curves. There are $d_{n, m}$ topologically inequivalent configurations, called arch configurations when $n-2 m=0$. We keep the same name for $m \neq 0$, writing $\operatorname{arch}_{m}$ configurations when precision is needed.

Motivated by this, we claim the following :

a) To each arch configuration $\alpha$ corresponds an extremal state $Z_{\alpha}$ in the following sense : the solution of the $n \mathrm{SLE}$ system with $Z \propto Z_{\alpha}$ can be defined up to a (possibly infinite) time, at which the growing curves have either paired together or joined the point at infinity and at that time the topology is that of the arch $\alpha$ with probability one.

b) One can decompose a general solution $Z$ of $i$ ), ii), iii) as a sum of

$$
\sum_{\alpha \in \operatorname{arch}_{m}} Z_{\alpha}
$$

c) The probability that a solution of the $n$ SLE system with auxiliary function $Z$ ends in arch configuration $\alpha$ is the ratio

$$
\frac{Z_{\alpha}\left(X_{1}, \cdots, X_{n}\right)}{Z\left(X_{1}, \cdots, X_{n}\right)}
$$

evaluated at the initial condition $\left(X_{1}, \cdots, X_{n}\right)$.

The first step toward a heuristic derivation of the above results will be to explain how to construct martingales - in particular martingales associated to interfaces - from statistical mechanics observables in a systematic way. But we start with a few comments. 


\section{First comments}

\subsection{Statistical mechanics interpretation}

To have a specific example in mind, think again of the Ising model at the critical temperature. Let $a$ be the lattice spacing.

First, put $n=2 m$ changes of boundary conditions from spins up to spins down and so on along the boundary at points $x_{1} / a, \cdots, x_{n} / a$. In the continuum limit when $a \rightarrow 0$ but $x_{1}, \cdots, x_{n}$ have a finite limit, the partition function behaves like a homogeneous function $Z\left(x_{1} / a, \cdots, x_{n} / a\right)$ of weight 0 (when both $a$ and the $x_{i}$ 's are rescaled) and CFT teaches us that $Z\left(x_{1}, \cdots, x_{n}\right)$ satisfies $\left.\left.i\right), i i\right)$, iii) for $n=2 m$. Then, if $\alpha$ is an arch system, $Z_{\alpha}$ should be (proportional to the continuum limit of) the partial partition function when the sum of Boltzmann weights is performed only over the interface configurations with topology $\alpha$.

To make generalized arch configurations, choose $n$ and $m$ with $n \geq 2 m$. Put $2 n-2 m$ changes of boundary conditions from spins up to spins down and so on along the boundary, $n$ at points $x_{1} / a, \cdots, x_{n} / a$ and $n-2 m$ at $y_{1} / a, \cdots, y_{n-2 m} / a$. Sum only over configurations where the interfaces do not joint two $y$-type points to each other. Take the continuum limit for the $x$ 's as before, but impose that all $y$ 's go to infinity and remain at a finite number of lattice spacings from each other. This is expected to lead again to a partition function $Z\left(x_{1} / a, \cdots, x_{n} / a\right)$ of weight 0 (when both $a$ and the $x_{i}$ 's are rescaled) and $Z\left(x_{1}, \cdots, x_{n}\right)$ satisfies $\left.\left.i\right), i i\right)$, iii) for the given $n$ and $m$. If $\alpha$ is an $\operatorname{arch}_{m}$ configuration, $Z_{\alpha}$ should be (proportional to the continuum limit of) the partial partition function when the sum of Boltzmann weights is performed only over the interface configurations with topology $\alpha$.

Note that the prefactor between the continuum limit finite part and the real partition function is a power of the lattice spacing. The power depends on $m$, so it is likely to be unphysical to use a non homogeneous $Z$ in the $n$ SLE system, mixing different values of $m$ for a fixed $n$. However, we shall later treat the example $n=2$ mixing $m=0$ and $m=1$ because it is illustrative despite the fact that it breaks scale invariance.

\subsection{SLE as a special case of $2 \mathrm{SLE}$}

For $n=2$ the solution of $i$ ), $i i)$, $i i i)$ with $m=1$ is elementary. Writing $x_{1}=a$ and $x_{2}=b$, one finds $Z \propto(b-a)^{(\kappa-6) / \kappa}$. Taking the first martingale to be a Brownian and the second one to be 0 , one retrieves the equations for SLE growing from point $a$ to point $b$ in the hydrodynamical normalization. Let us recall briefly why. 
We start from SLE from 0 to $\infty$. The basic principle of conformal invariance makes the passage from this special case to the case when SLE goes from point $a$ to point $b$ on $\mathbb{H}$ a routine task. If $u$ is any linear fractional transformation (i.e. any conformal transformation) from $\mathbb{H}$ to itself mapping 0 to $a$ and $\infty$ to $b$, the image of the SLE trace or hull from 0 to $\infty$ by $u$ is by definition an SLE trace from $a$ to $b$ and this is measure preserving. The new uniformizing map is $h_{t}=u \circ g_{t} \circ u^{-1}$ and it is readily checked that $\frac{\mathrm{d} h_{t}}{\mathrm{~d} t}$ is a rational function of $h_{t}$ whose precise form can be easily computed but does not concern us.

Let us just mention that this rational function is regular everywhere (infinity included) except for a simple pole at $h_{t}=u\left(\xi_{t}\right)$ and has a third order zero at $h_{t}=u(\infty)=b$. So the map $h_{t}$ is normalized in such a way that $h_{t}(b+\varepsilon)=b+\varepsilon+O\left(\varepsilon^{3}\right)$, which is not the hydrodynamic normalization.

But if $v_{t}$ is any linear fractional transformation, $v_{t} \circ g_{t} \circ u^{-1}$ describes the same trace as $h_{t}=u \circ g_{t} \circ u^{-1}$. As long as the trace does not separate $b$ from $\infty$, i.e. as long as the trace has not hit the real axis in the segment ]$b, \infty\left[\right.$, i.e. as long as $\infty$ is not in the hull, $v_{t}$ can be adjusted in such a way that $\tilde{h}_{t} \equiv v_{t} \circ g_{t} \circ u^{-1}$ is normalized hydrodynamically. Then $\mathrm{d} \tilde{h}_{t} / \mathrm{d} t$ is a function of $\tilde{h}_{t}$ which is regular everywhere but for a single pole and vanishes at infinity, i.e. one can write $\mathrm{d} \tilde{h}_{t} / \mathrm{d} t=2 \mu_{t} /\left(\tilde{h}_{t}-\alpha_{t}\right)$. The following computation is typical of the manipulations made with SLE (see e.g. [4]). Write $\left(g_{t} \circ u^{-1}\right)(z)=w$ an compute from the definition

$$
\frac{\mathrm{d} \tilde{h}_{t}}{\mathrm{~d} t}(z)=\frac{\mathrm{d} v_{t}}{\mathrm{~d} t}(w)+v_{t}^{\prime}(w) \frac{2}{w-\xi_{t}} .
$$

Comparison gives

$$
\frac{\mathrm{d} v_{t}}{\mathrm{~d} t}(w)=\frac{2 \mu_{t}}{v_{t}(w)-\alpha_{t}}-\frac{2 v_{t}^{\prime}(w)}{w-\xi_{t}}
$$

But $v_{t}$ is regular at $w=\xi_{t}$ from which one infers that $v_{t}\left(\xi_{t}\right)=\alpha_{t}$ (the poles in the two terms are at the same point) and $\mu_{t}=v_{t}^{\prime}\left(\alpha_{t}\right)^{2}$ (the two residues add to 0$)$. Going one step further in the expansion close to $\xi_{t}$ yields $\frac{\mathrm{d} v_{t}}{\mathrm{~d} t}\left(\xi_{t}\right)=$ $-3 v_{t}^{\prime \prime}\left(\xi_{t}\right)$. Ito's formula gives $\mathrm{d} \alpha_{t}=-3 v_{t}^{\prime \prime}\left(\xi_{t}\right) \mathrm{d} t+v_{t}^{\prime}\left(\xi_{t}\right) \mathrm{d} \xi_{t}+\frac{\kappa}{2} v_{t}^{\prime \prime}\left(\xi_{t}\right) \mathrm{d} t$. So the time change $\mu_{t} \mathrm{~d} t=\mathrm{d} s$ together with the definition $\mathrm{d} \chi_{s}=v_{t}^{\prime}\left(\xi_{t}\right) \mathrm{d} \xi_{t}$ yields

$$
\mathrm{d} \alpha_{t}(s)=\mathrm{d} \chi_{s}+(\kappa-6) \frac{v_{t}^{\prime \prime}\left(\xi_{t}\right)}{2 v_{t}^{\prime}\left(\xi_{t}\right)^{2}} \mathrm{~d} s .
$$

But $v_{t}^{\prime \prime}(w) / v_{t}^{\prime}(w)^{2}=2 /\left(v_{t}(w)-v_{t}(\infty)\right)$ because $v_{t}$ is a linear fractional transformation. Finally, setting $\tilde{h}_{t(s)} \equiv f_{s}, \tilde{h}_{t(s)}(b)=v_{t(s)}(\infty) \equiv B_{s}$ and 
$v_{t(s)}\left(\xi_{t(s)}\right)=\alpha_{t(s)} \equiv A_{s}$ we can summarize

$$
\frac{\mathrm{d} f_{s}}{\mathrm{~d} s}=\frac{2}{f_{s}-A_{s}}, \quad \frac{\mathrm{d} B_{s}}{\mathrm{~d} s}=\frac{2}{B_{s}-A_{s}}, \quad \mathrm{~d} A_{s}=\mathrm{d} \chi_{s}+(\kappa-6) \frac{\mathrm{d} s}{A_{s}-B_{s}},
$$

where $\chi_{s}$ is a Brownian motion with quadratic variation $\kappa s, f_{0}=i d, A_{0}=a$, $B_{0}=b$. Thus chordal SLE from $a$ to $b$ in the hydrodynamical normalization is indeed a special case of 2SLE.

The above equations are also a special case of $\operatorname{SLE}(\kappa, \rho)(\rho=\kappa-6)$, but it should be clear that our general proposal goes in a different direction.

As already mentioned, the description of chordal SLE from $a$ to $b$ in the hydrodynamical normalization in fact coincides with chordal SLE from $a$ to $b$ only up to the first time $b$ is separated from $\infty$ by the trace. This time is infinite for $\kappa \leq 4$, but it is finite with probability 1 for $4<\kappa<8$. The most obvious case is $\kappa=6$. The equation is nothing but the usual chordal $\mathrm{SLE}_{6}$ ending at infinity, a consequence of locality (in the SLE sense, not in the quantum field theory sense used later). At that time, the real chordal SLE from $a$ to $b$ swallows $\infty$, whereas the hydrodynamically normalized version swallows $b$. The solution to this problem is to use conformal invariance and restart the process again in the correct domain at the time when $b$ and $\infty$ get separated by the trace. But this is not coded in the equations.

\subsection{Making sense}

The previous example should serve as a warning. Some serious mathematical work may have to be done even to make sense of our conjectures, let alone prove their correctness. The problems might be of different natures for $\kappa \leq 4$ and $4<\kappa<8$. We content with the following naive remarks. One of the problems is that the arches do not have to close at the same time. It may even happen that one of the growing curves touches the real line or another curve in such a way that the upper half plane is split in two domains and the one which is swallowed contains some of the growing curves.

Our putative description of $n$ SLE processes can be valid in this form only up the realization of such an event. The first thing to check should be that the event is realized with a probability obtained by summing $Z_{\alpha} / Z$ over all $\alpha$ 's corresponding to compatible configurations (see figure 1). In particular, the connected component of $\infty$ should contain at least $n_{\infty} \geq m-1$ curves for consistency, but that's not an obvious property of our proposal.

Consider the fate of the connected component of $\infty$. If $n_{1}-m$ is even, conformal invariance suggests to continue the Loewner evolution simply by suppressing the points that have been swallowed, i.e. for the $n_{1}$ remaining 

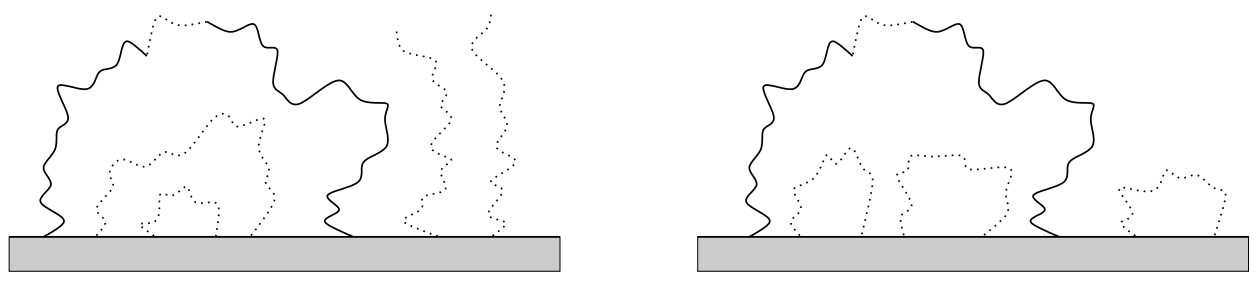

Figure 1: The probability of closing of an arch should be obtained by summing $Z_{\alpha} / Z$ over all $\alpha$ 's corresponding to compatible configurations. Two compatible configurations are portrayed in the figure.

points. If $n_{1}-m$ is odd, the same should be done, but the image of the point at which one interface has made a bridge should be included as a starting point for the continuation of the evolution. Preferably, the function $Z$ for this new multiSLE system should not be adjusted by hand to make our conjectures correct, but should appear as a natural limit. We shall make comments on this and give concrete illustrations later.

For the component that is swallowed, one can use conformal invariance again to change the normalization of the Loewner map in such a way that this component is the one that survives and then restart a new multiSLE for the appropriate number of points. This procedure may have to be iterated.

Note also that our conjectures for arch probabilities do not involve any details on the martingales $M_{t}^{(i)}$. Indeed, we expect that there is some robustness. But the precise criteria are beyond our understanding.

\subsection{A few martingales for $n$ SLEs}

Our heuristic derivation of the $n$ SLE system will in particular show that if $\tilde{Z}$ also solves $i$ ), $i i$ ), iii) (even relaxing positivity), the quotient

$$
\frac{\tilde{Z}\left(X_{t}^{(1)}, \cdots, X_{t}^{(n)}\right)}{Z\left(X_{t}^{(1)}, \cdots, X_{t}^{(n)}\right)}
$$

is a local martingale. This can be proved directly using Ito's formula.

In particular,

$$
\frac{Z_{\alpha}\left(X_{t}^{(1)}, \cdots, X_{t}^{(n)}\right)}{Z\left(X_{t}^{(1)}, \cdots, X_{t}^{(n)}\right)}
$$

is a local martingale bounded by 1 , hence a martingale. On the other hand, a standard argument shows that if $P_{\alpha}$ is the probability that the system ends in a definite arch configuration $\alpha$ (once one has been able to make sense of 
it) $P_{\alpha}\left(X_{t}^{(1)}, \cdots, X_{t}^{(n)}\right)$ is a martingale. This is an encouraging sign. To get a full proof, one would need to analyze the behavior of $Z_{\alpha}\left(X_{t}^{(1)}, \cdots, X_{t}^{(n)}\right)$ when one arch closes, or when one growing curve cuts the system in two, to get recursively a formula that looks heuristically like

$$
\frac{Z_{\alpha}\left(X_{t}^{(1)}, \cdots, X_{t}^{(n)}\right)}{Z\left(X_{t}^{(1)}, \cdots, X_{t}^{(n)}\right)} \sim \delta_{\alpha, \alpha^{\prime}}
$$

if the system forms asymptotically the arch system $\alpha^{\prime}$ at large $s$. Such a formula rests on properties of $Z_{\alpha}\left(x_{1}, \cdots, x_{n}\right)$ when some points come close together in a way reminiscent to the formation of arch $\alpha^{\prime}: Z_{\alpha^{\prime}}\left(x_{1}, \cdots, x_{n}\right)$ should dominate all $Z_{\alpha}$ 's, $\alpha \neq \alpha^{\prime}$ in such circumstances. In section 8 we shall use this to expand explicitly the $Z_{\alpha}$ 's in a basis of solutions to $i$ ), ii), iii) which is familiar from CFT, very explicitly at least for small $n$.

\subsection{Classical limit}

Our proposal for $n$ SLE has a non trivial classical limit at $\kappa \rightarrow 0^{+}$. The martingales $M_{t}^{(i)}$ vanish in this limit, but the $q_{t}^{(i)}$ remain arbitrary increasing functions. The function $Z$ does not have a limit, but the $U_{i} \equiv \kappa \partial_{x_{i}} \log Z$ do. They are kind of Ricatti variables for which the equations read

$$
\frac{1}{2}\left(\partial_{x_{i}} U_{i}+\frac{U_{i}^{2}}{\kappa}\right)+2 \sum_{j \neq i}\left(\frac{1}{x_{j}-x_{i}} \frac{U_{i}}{\kappa}-\frac{6-\kappa}{2 \kappa} \frac{1}{\left(x_{j}-x_{i}\right)^{2}}\right)=0,
$$

which have a limit when $\kappa \rightarrow 0^{+}$, comparable to the classical limit of a Schroedinger equation. To summarize, the classical limit is

$$
\begin{gathered}
\mathrm{d} f_{t}(z)=\sum_{i} \frac{2 \mathrm{~d} q_{t}^{(i)}}{f_{t}(z)-X_{t}^{(i)}} . \\
\mathrm{d} X_{t}^{(i)}=U_{i}\left(X_{t}^{(1)}, \cdots, X_{t}^{(n)}\right) \mathrm{d} q_{t}^{(i)}+\sum_{j \neq i} \frac{2 \mathrm{~d} q_{t}^{(j)}}{X_{t}^{(i)}-X_{t}^{(j)}} .
\end{gathered}
$$

where the auxiliary functions $U_{i}\left(x_{1}, \cdots, x_{n}\right)$ are homogeneous functions of degree -1 which satisfy $\partial_{x_{i}} U_{j}=\partial_{x_{j}} U_{i}$ and

$$
\frac{1}{2} U_{i}^{2}+2 \sum_{j \neq i}\left(\frac{1}{x_{j}-x_{i}} U_{i}-\frac{3}{\left(x_{j}-x_{i}\right)^{2}}\right)=0 .
$$




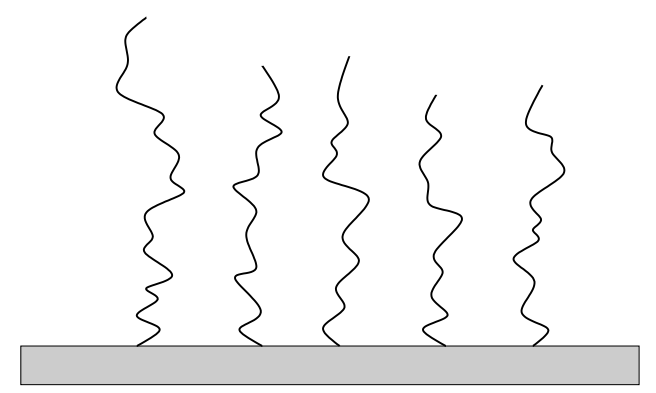

Figure 2: The factorisable $Z$ leads to a very simple geometry. This case has been suggested previously with a slightly different approach.

It is not too surprising that the differential equations for $Z$ have become algebraic equations for the $U_{i}$ 's, so that the space of solutions which was a connected manifold for $\kappa \neq 0$ concentrates on a finite number of points in the classical limit. The classical system, maybe with an educated guess for the $q_{t}^{(i)}$ s, could be interesting for its own sake.

\subsection{Relations with other work}

Several processes involving several growing curves have appeared in the literature.

The first proposal was made by Cardy [15]. It can be formally obtained from ours by forgetting the conditions $i$ ), ii), iii) and choosing a constant $Z$. The corresponding processes are interesting, but the relationship with interfaces in statistical mechanics and CFT is unclear for us.

Dubédat [16] has derived a general criterion he calls commutativity to constrain the class of processes that could possibly be related to interfaces. Our proposal satisfies commutativity so they can be viewed as a special case satisfying other relevant physical constraints. Dubédat also came with a special solution of commutativity. It corresponds to the case $m=0$ in our language. Then the space of solutions has dimension $d_{n, 0}=1$ and the corresponding partition function is elementary:

$$
Z \propto \prod_{i<j}\left(x_{j}-x_{i}\right)^{2 / \kappa}
$$

A single arch topology is possible, all interfaces converge to $\infty$, see figure 2. Maybe this is a good reason to call this case chordal $n \mathrm{SLE}$. 


\section{$5 \quad$ CFT background}

There was never any doubt that SLEs are related to conformal field theories. The original approach [7, 12, 11, 14] used the operator formalism because if yields naturally martingale generating functions. Here, we use the correlator approach for a change. We restrict the presentation to a bare minimum, referring the newcomer to the many articles, reviews and books on the subject (18, 17]). The reader who knows too little or too much about CFT can profitably skip this section.

Observables in CFT can be classified according to their behavior under conformal maps. Local observables in quantum field theory are called fields. For instance, in the Ising model, on an arbitrary (discrete) domain, the average value of a product of spins on different (well separated) sites can be considered. Taking the continuum limit at the critical point, we expect that on arbitrary domains $D$ there is a local observable, the spin. The product of two spins at nearest neighbor points corresponds to the energy operator. In the continuum limit, this will also lead to a local operator. In this limit, the lattice spacing has disappeared and one can expect a definite (but nontrivial) relationship between the energy operator and the product of two spin fields close to each other. As on the lattice the product of two spins at the same point is 1 , we can expect that the identity observable also appears in such a product at short distances. Local fields come in two types, bulk fields whose argument runs over $D$ and boundary fields whose argument runs over $\partial D$. In this paper, we shall not need bulk fields so we leave them aside.

The simplest conformal transformations in the upper-half plane are real dilatations and boundary fields can be classified accordingly. It is customary to write $\varphi_{\delta}(x)$ to indicate that in a real dilatation by a factor $\lambda$ the field $\varphi_{\delta}(x)$ picks a factor $\lambda^{\delta}$. By a locality argument, boundary fields in a general domain $D$ (not invariant under dilatations) can still be classified by the same quantum number. The number $\delta$ is called the conformal weight of $\varphi_{\delta}$.

There are interesting situations in which (due to degeneracies) the action of dilatations cannot be diagonalized, leading to so called logarithmic CFT. While this more general setting is likely to be relevant for several aspects of SLE, we shall not need it in what follows.

Under general conformal transformations, the simplest objects in CFT are so called primary fields. Their behavior is dictated by the simplest generalization of what happens under dilatations. Suppose $\varphi_{\delta_{1}}, \cdots \varphi_{\delta_{n}}$ are boundary primary fields of weights $\delta_{1}, \cdots, \delta_{n}$. If $f$ is a conformal map from 
domain $D$ to a domain $D^{\prime}$, CFT postulates that

$$
\left\langle\prod_{j=1}^{n} \varphi_{\delta_{j}}\left(x_{j}\right)\right\rangle^{D}=\left\langle\prod_{j=1}^{n} \varphi_{\delta_{j}}\left(f\left(x_{j}\right)\right)\right\rangle^{f(D)} \prod_{j=1}^{n}\left|f^{\prime}\left(x_{j}\right)\right|^{\delta_{j}} .
$$

Symbolically, this can be written $f: \varphi_{\delta}(x) \rightarrow \varphi_{\delta}(f(x))\left|f^{\prime}(x)\right|^{\delta}$. It is interesting to make a comparison of these axioms with the previous computations relating chordal SLE from 0 to $\infty$ to chordal SLE from $a$ to $b$ in several normalizations. This also involved pure kinematics.

As usual in quantum field theory, to a symmetry corresponds an observable implementing it. In CFT, this leads to the stress tensor $T(z)$ whose conservation equation reduces to holomorphicity. The fact that conformal transformations are pure kinematics translates into the fact that insertions of $T$ in known correlation functions can be carried automatically, at least recursively. The behavior of $T(z)$ under conformal transformations can be written as $f: T(z) \rightarrow T(f(z)) f^{\prime}(z)^{2}+c / 12 S f(z)$ where $S f \equiv\left(f^{\prime \prime} / f^{\prime}\right)^{\prime}-1 / 2\left(f^{\prime \prime} / f^{\prime}\right)^{2}$ is the Schwarzian derivative and $c$ is a conformal anomaly, a number which is the most important numerical characteristic of a CFT. When $c=0, T$ is be a $(2,0)$ primary field i.e. an holomorphic quadratic differential. When a (smooth) boundary is present, the Schwarz reflection principle allows to extend $T$ by holomorphicity. Holomorphicity also implies that if $O$ is any local (bulk or boundary) observable at point $z \in D$ and $v$ is vector field meromorphic close to $z$, the contour integral $L_{v} O \equiv \oint_{z} d w v(w) T(w) O$ along an infinitesimal contour around $z$ oriented counterclockwise is again a local field at $z$, corresponding to the infinitesimal variation of $O$ under the map $f(w)=w+\varepsilon v(w)$. It is customary to write $L_{n}$ for $v(w)=w^{n+1}$. It is one of the postulates of CFT that all local fields can be obtained as descendants of primaries, i.e. by applying this construction recursively starting from primaries. The correlation functions of descendant fields are obtained in a routine way from correlations of the primaries. But descendant fields do not transform homogeneously.

When $v$ is holomorphic at $x, L_{v} O$ is a familiar object. For instance, if $\varphi_{\delta}$ is a primary boundary field, one checks readily that $L_{n} \varphi_{\delta}=0$ for $n \geq 1$, $L_{0} \varphi_{\delta}=\delta \varphi_{\delta}$ and $L_{-1} \varphi_{\delta}=\Re e\left[\partial_{x} \varphi_{\delta}\right]$. The other descendants are in general more involved, but by definition the stress tensor $T=L_{-2} I d$ is the simplest descendant of the identity $I d$. It does indeed not transform homogeneously.

A primary field and its descendants form what is called a conformal family. Not all linear combinations of primaries and descendants need to be independent. The simplest example is the identity observable, which is primary with weight 0 and whose derivative along the boundary vanishes 
identically ${ }^{4}$. By contour deformation, this leads to translation invariance of correlation functions when $D$ has translation symmetry.

The next example in order of complexity is of utmost importance for the rest of this paper. If $(2 h+1) c=2 h(8 h-5)$, the field

$$
-2(2 h+1) L_{-2} \varphi_{h}+3 L_{-1}^{2} \varphi_{h}
$$

is again a primary, i.e. it transforms homogeneously under conformal maps. In this case, consistent CFTs can be constructed for which it vanishes identically. This puts further constraints on correlators.

For example, when $D$ is the upper half plane, so that the Schwarz principle extends $T$ to the full plane, the contour for $L_{-2}$ can be deformed and shrunken at infinity. Then, for an arbitrary boundary primary correlator one has

$$
\begin{aligned}
\left(\frac{3}{2(2 h+1)} \partial_{x}^{2}+\sum_{\alpha=1}^{l}\left[\frac{1}{y_{\alpha}-x} \partial_{y_{\alpha}}\right.\right. & \left.\left.-\frac{\delta_{\alpha}}{\left(y_{\alpha}-x\right)^{2}}\right]\right) \\
& \left\langle\varphi_{\delta}(\infty) \prod_{\alpha=1}^{l} \varphi_{\delta_{\alpha}}\left(y_{\alpha}\right) \varphi_{h}(x)\right\rangle=0 .
\end{aligned}
$$

It is customary to call this type of equation a null-vector equation.

Note that the primary field of weight $\delta$ sitting at $\infty$ has led to no contribution in this differential equation. Working the other way round, this equation valid for an arbitrary number of boundary primary fields with arbitrary weights characterizes the field $\varphi_{h}$ and the relation between $h$ and the central charge $c$.

The case of three points correlators is instructive. Global conformal invariance implies that

$$
\left\langle\varphi_{\delta}(y) \varphi_{\delta^{\prime}}\left(y^{\prime}\right) \varphi_{h}(x)\right\rangle \propto\left|y-y^{\prime}\right|^{h-\delta-\delta^{\prime}}|x-y|^{\delta^{\prime}-h-\delta}\left|y^{\prime}-x\right|^{\delta-\delta^{\prime}-h} .
$$

The proportionality constant might depend on the cyclic ordering of the three points. But if the differential equation for $\varphi_{h}$ is used, a further constraint appears. The three point function can be non vanishing only if

$$
3\left(\delta-\delta^{\prime}\right)^{2}-(2 h+1)\left(\delta+\delta^{\prime}\right)=h(h-1) .
$$

This computation has a dual interpretation : consider a correlation function with any number of fields, among them a $\varphi_{\delta}(y)$ and a $\varphi_{h}(x)$. If $x$ and $y$

\footnotetext{
${ }^{4}$ For other primary fields with the same weight if any, this does not have to be true.
} 
come very close to each other they can be replaced by an expansion in terms of local fields. This is called fusion. Several conformal families can appear in such an expansion, but within a conformal family, the most singular contribution is always from a primary. This argument applies even if $c$ and $h$ are arbitrary. But suppose they are related as above and the differential equation eq. (5) is valid. This equation is singular at $x=y$ and at leading order the dominant balance leads to an equation where the other points are spectators. One finds that the only conformal families that can appear are the ones whose conformal weight $\delta^{\prime}$ satisfies the fusion rule.

This is enough CFT background for the rest of this paper. We are now in position to give the heuristic argument that leads to our main claims.

\section{Martingales from statistical mechanics}

The purpose of this section is to emphasize the intimate connection between the basic rules of statistical mechanics and martingales. The connection is somehow tautological, because statistical mechanics works with partition functions, i.e. unnormalized probability distributions, all the time. In the discrete setting, this makes conditional expectations a totally transparent operation that one performs without thinking and even without giving it a name. But the following argument is, despite its simplicity and its abstract nonsense flavor, the crucial observation that allows us to relate interfaces in conformally invariant statistical mechanics to SLEs.

\subsection{Tautological martingales}

Consider a model of statistical mechanics with a finite but arbitrarily large set of possible states $S$. Usually one starts with models defined on finite grid domains so $\# S<\infty$ is natural. To each state $s \in S$ we associate a Boltzmann weight ${ }^{5} w(s)$. The partition function is $Z=\sum_{s \in S} w(s)$ so that it normalizes the Boltzmann weights to probabilities, $\mathbb{P}\{s\}=\frac{w(s)}{Z}$. Since $S$ is finite, we can use the power set $\mathcal{P}(S)=\{U: U \subset S\}$ as a sigma algebra. The expected value of a random variable $\mathcal{O}: S \rightarrow \mathbb{C}$ is denoted by $\mathbb{E}[\mathcal{O}]=\langle\mathcal{O}\rangle=\frac{1}{Z} \sum_{s \in S} \mathcal{O}(s) w(s)$.

Note that if $\left(S_{\alpha}\right)_{\alpha \in I}$ is a collection of disjoint subsets of $S$ such that $\cup_{\alpha \in I} S_{\alpha}=S$, then the collection of all unions $\mathcal{F}=\left\{\cup_{\alpha \in I^{\prime}} S_{\alpha}: I^{\prime} \subset I\right\}$ is a

\footnotetext{
${ }^{5}$ Usually the Boltzmann weight is related to the energy $H(s)$ of the state $s$ through $w(s)=\exp (-\beta H(s))$, where $\beta$ is the inverse temperature (a Lagrangian multiplier related to temperature, anyway).
} 
sigma algebra on $S$. Conversely, since $S$ is finite, any sigma algebra $\mathcal{F}$ on $S$ is of this type.

Consider a filtration, that is an increasing family $\left(\mathcal{F}_{t}\right)_{t \geq 0}$ of sigma algebras $\{\emptyset, S\} \subset \mathcal{F}_{s} \subset \mathcal{F}_{t} \subset \mathcal{P}(S)$ for all $0 \leq s<t$. Denote the corresponding collections of disjoint sets by $\left(S_{\alpha}^{(t)}\right)_{\alpha \in I_{t}}$ and define the partial partition function $Z_{\alpha}^{(t)} \equiv \sum_{s \in S_{\alpha}^{(t)}} w(s)$. The conditional expectation values

$$
\begin{aligned}
\langle\mathcal{O}\rangle_{t} & \equiv \mathbb{E}\left[\mathcal{O} \mid \mathcal{F}_{t}\right]=\sum_{\alpha \in I_{t}} \frac{\sum_{s \in S_{\alpha}^{(t)}} \mathcal{O}(s) w(s)}{\sum_{s \in S_{\alpha}^{(t)}} w(s)} \mathbf{1}_{S_{\alpha}^{(t)}} \\
& =\sum_{\alpha \in I_{t}}\left(\frac{1}{Z_{\alpha}^{(t)}} \sum_{s \in S_{\alpha}^{(t)}} \mathcal{O}(s) w(s)\right) \mathbf{1}_{S_{\alpha}^{(t)}}
\end{aligned}
$$

are martingales by definition: for $s<t$ we have

$$
\mathbb{E}\left[\mathbb{E}\left[\mathcal{O} \mid \mathcal{F}_{t}\right] \mid \mathcal{F}_{s}\right]=\mathbb{E}\left[\mathcal{O} \mid \mathcal{F}_{s}\right]
$$

Notice that the probability of the event $S_{\alpha}^{(t)}$ is conveniently $\mathbb{P}\left[S_{\alpha}^{(t)}\right]=Z_{\alpha}^{(t)} / Z$.

Suppose that the model is defined in a domain $D \subset \mathbb{C}$ and that there are interfaces in the model. Parametrize portions of these interfaces touching the boundary by an arbitrary "time" parameter $t$ in such a way that $n$ paths $\gamma_{t}^{(i)}, i=1, \cdots, n$ (which are pieces of the random interfaces) emerge from the boundary at $t=0$ and are disjoint at least when $t$ is small enough, see figure 3. To avoid confusion we write the time parameter $t$ now as a subscript and continue to indicate the dependence of $s \in S$ by parenthesis, so $t \mapsto \gamma_{t}^{(i)}(s)$ is a parametrization of the $i^{\text {th }}$ piece of interface if the system is at state $s$. Then we can consider the natural filtration of the interface by taking $\mathcal{F}_{t}=\sigma\left(\gamma_{t^{\prime}}^{(i)}: 0 \leq t^{\prime} \leq t, i=1, \cdots, n\right)$ to be the sigma algebra generated by the random variables $\gamma_{t^{\prime}}^{(i)}$ up to time $t$.

The boundary conditions of the model are often such that conditioning on the $\gamma_{[0, t]}^{(i)}$, is the same as considering the model in a smaller domain (a part of the interface removed) but with same type of boundary conditions. Of course the position at which the new interface should start is where the original interface would have continued, that is the $\gamma_{t}^{(i)}$, s. Let $D_{t}$ be the domain $D$ with the $\gamma_{j 0, t]}^{(i)}$ removed.

The starting point of the next section is the input of conformal invariance in this setup. 


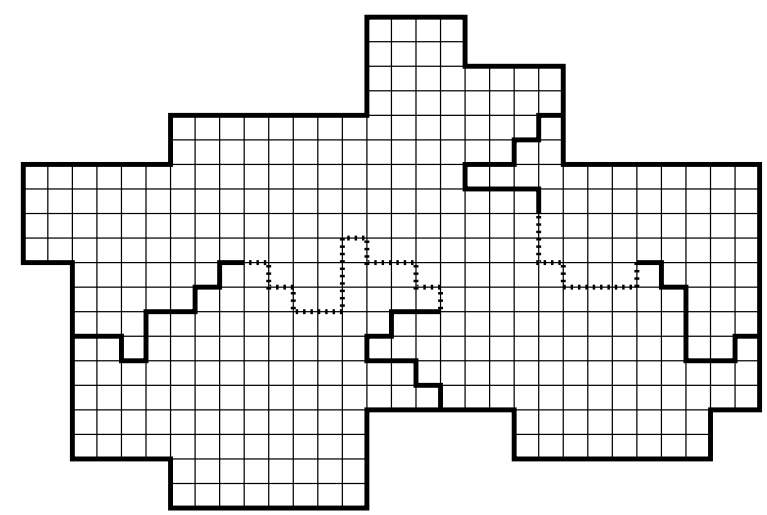

Figure 3: A discrete statistical mechanics model with portions of interfaces specified.

\subsection{Simplifying tautological martingales}

We start from the situation and notations at the end of the previous section. If in addition we are considering a model at its critical point, then the continuum limit may be described by a conformal field theory. At least for a wide class of natural observables $\mathcal{O}$, the expectation values become $\mathrm{CFT}$ correlation functions in the domain $D$ of the model

$$
\langle\mathcal{O}\rangle=\frac{\sum_{s \in S} \mathcal{O}(s) w(s)}{Z} \longrightarrow \frac{\langle\mathcal{O}\rangle_{D}^{\mathrm{CFT}, \text { b.c. }}}{\langle\mathbf{1}\rangle_{D}^{\mathrm{CFT}, \text { b.c. }}}
$$

We need to write the correlation function of identity (proportional to $Z$ ) in the denominator because the boundary conditions (b.c.) of the model may already have led to insertions of boundary changing operators that we have not mentioned explicitly.

The closed martingales become

$$
\langle\mathcal{O}\rangle_{t}=\sum_{\alpha \in I_{t}} \frac{1}{Z_{\alpha}^{(t)}} \sum_{s \in S_{\alpha}^{(t)}} \mathcal{O}(s) w(s) \mathbf{1}_{S_{\alpha}^{(t)}} \longrightarrow \frac{\langle\mathcal{O}\rangle_{D_{t}}^{\mathrm{CFT}, \text { b.c. }}}{\langle\mathbf{1}\rangle_{D_{t}}^{\mathrm{CFT}, \text { b.c. }}}
$$

where in the continuum limit $D_{t}$ might be $D$ with hulls (and not only traces) removed.

For certain (but not all) observables, $\langle\mathcal{O}\rangle$ is computing a probability, which in a conformal field theory ought to be conformally invariant. But $\langle\mathcal{O}\rangle$ is written as a quotient, and this means that the numerator and denominator should transform homogeneously (and with the same factor) under 
conformal transformations. In particular, the denominator should transform homogeneously. This means that $\langle\mathbf{1}\rangle_{D}^{C F T, \text { b.c. }}$ - which depends on the position of the boundary condition changes - behaves like a product of boundary primary fields. Then, by locality, for any $\mathcal{O}$, the transformation of the numerator under conformal maps will split in two pieces: one containing the transformations of $\mathcal{O}$ and the other one canceling with the factor in the denominator. So we infer the existence in the CFT of a primary boundary field, denoted by $\psi(x)$ in what follows, which implements boundary condition changes at which interfaces anchor. Hence we may write

$$
\langle\mathbf{1}\rangle_{D}^{\mathrm{CFT}, \text { b.c. }}=\left\langle\psi\left(X^{(1)}\right) \cdots \psi\left(X^{(n)}\right)\right\rangle_{D}^{\mathrm{CFT}}
$$

and

$$
\langle\mathcal{O}\rangle_{D}^{\mathrm{CFT}, \text { b.c. }}=\left\langle\mathcal{O} \psi\left(X^{(1)}\right) \cdots \psi\left(X^{(n)}\right)\right\rangle_{D}^{\mathrm{CFT}} .
$$

As will become clear later, there might also be one further boundary operator anchoring several interfaces. We do not mention it explicitly here because it will sit at a point which will not be affected by the conformal transformations that we use.

Write the transformation of the observable $\mathcal{O}$ as $f: \mathcal{O} \rightarrow{ }^{f_{\mathcal{O}}}$ under a conformal map. Denote by $f_{t}$ a conformal representation $f_{t}: D_{t} \rightarrow D$ and write $f\left(\gamma_{t}^{(i)}\right) \equiv X_{t}^{(i)}$. The expression for the closed martingale $\langle\mathcal{O}\rangle_{t}$ can now be simplified further

$$
\langle\mathcal{O}\rangle_{t} \longrightarrow \frac{\left\langle{ }^{f_{t}} \mathcal{O} \psi\left(X_{t}^{(1)}\right) \cdots \psi\left(X_{t}^{(1)}\right)\right\rangle_{D}^{\mathrm{CFT}}}{\left\langle\psi\left(X_{t}^{(1)}\right) \cdots \psi\left(X_{t}^{(1)}\right)\right\rangle_{D}^{\mathrm{CFT}}} .
$$

The Jacobians coming from the transformations of the boundary changing primary field $\psi$ have canceled in the numerator and denominator. The explicit value of the conformal weight of $\psi$ does not appear in this formula.

Of course, we have cheated. For the actual map $f_{t}$ which is singular at the $\gamma_{t}^{(i)}$ 's these Jacobians are infinite. A more proper "derivation" would go through a regularization but locality should ensure that the naive formula remains valid when the regularization is removed. Eq.(6) is the starting point of our analysis.

\section{Derivation of the proposal}

The heuristics we follow is to describe a growth process of interfaces by a Loewner chain $f_{t}$ compatible with conformal invariance in that the right hand side of eq.(6) is a martingale. 


\subsection{The three ingredients}

Loewner chain: If we use the upper half plane as a domain, $D=\mathbb{H}$, and impose the hydrodynamic normalization, the equation for $f_{t}$ has to be of the form

$$
\mathrm{d} f_{t}(z)=\sum_{i} \frac{2 \mathrm{~d} q_{t}^{(i)}}{f_{t}(z)-X_{t}^{(i)}}
$$

for some processes $X_{t}^{(i)}, i=1, \ldots, n$. The initial condition is $f_{0}(z)=z$.

Interfaces grow independently of each other on very short time scales: Schramm's argument deals with the case of a single point. We expect that on very short time scales the growth processes do not feel each other and Schramm's argument is still valid, so that $\mathrm{d} X_{t}^{(i)}=\mathrm{d} M_{t}^{(i)}+F_{t}^{(i)}$ where the $M_{t}^{(i)}$ 's are $n$ (continuous,local) martingales with quadratic variation $\kappa q_{t}^{(i)}$ and vanishing cross variation and $F_{t}^{(i)}$ is a drift term.

The martingale property fixes the drift term: The drift term will be computed by imposing the martingale condition on $\langle\mathcal{O}\rangle_{t}$ when $\mathcal{O}$ is a product of an arbitrary number $l$ of boundary primary fields $\mathcal{O}=\prod_{\alpha=1}^{l} \varphi_{\delta_{\alpha}}\left(Y^{(\alpha)}\right)$. The insertion points are away from the boundary changing operators and $f_{t}$ is regular with positive derivative there. Substitution of ${ }^{f_{t}} \mathcal{O}$ in formula (6) yields

$$
\left\langle\prod_{\alpha=1}^{l} \varphi_{\delta_{\alpha}}\left(Y^{(\alpha)}\right)\right\rangle_{t}=\frac{\left\langle\prod_{\alpha=1}^{l} \varphi_{\delta_{\alpha}}\left(f_{t}\left(Y^{(\alpha)}\right)\right) \prod_{i=1}^{n} \psi\left(X_{t}^{(i)}\right)\right\rangle_{D}^{\mathrm{CFT}}}{\left\langle\prod_{i=1}^{n} \psi\left(X_{t}^{(i)}\right)\right\rangle_{D}^{\mathrm{CFT}}} \prod_{\alpha=1}^{l} f_{t}^{\prime}\left(Y^{(\alpha)}\right)^{\delta_{\alpha}} .
$$

\subsection{Computation of the Ito derivative of $\left\langle\prod_{\alpha=1}^{l} \varphi_{\delta_{\alpha}}\left(Y^{(\alpha)}\right)\right\rangle_{t}$}

In formula (7), denote respectively by $Z_{t}^{\varphi}, Z_{t}$ and $J_{t}^{\varphi}$ the numerator, denominator and Jacobian factor on the right hand side.

It is useful to break the computation of $\mathrm{d}\left\langle\prod_{\alpha=1}^{l} \varphi_{\delta_{\alpha}}\left(Y^{(\alpha)}\right)\right\rangle_{t}$ in several steps.

- Preliminaries.

Ito's formula for the $\psi$ 's gives

$$
\mathrm{d} \psi\left(X_{t}^{(i)}\right)=\psi^{\prime}\left(X_{t}^{(i)}\right)\left(\mathrm{d} M_{t}^{(i)}+F_{t}^{(i)}\right)+\frac{\kappa}{2} \psi^{\prime \prime}\left(X_{t}^{(i)}\right) \mathrm{d} q_{t}^{(i)} .
$$


Using the Loewner chain for $f_{t}(z)$ and its derivative with respect to $z$, one checks that

$\mathrm{d}\left(\varphi_{\delta}\left(f_{t}(Y)\right) f_{t}^{\prime}(Y)^{\delta}\right)=f_{t}^{\prime}(Y)^{\delta} \sum_{i} 2 \mathrm{~d} q_{t}^{(i)}\left(\frac{\varphi_{\delta}^{\prime}\left(f_{t}(Y)\right)}{f_{t}(Y)-X_{t}^{(i)}}-\frac{\delta \varphi_{\delta}\left(f_{t}(Y)\right)}{\left(f_{t}(Y)-X_{t}^{(i)}\right)^{2}}\right)$.

- The Ito derivative of $Z_{t}^{\varphi} J_{t}^{\varphi}$.

The time $t$ being given, we can simplify the notation. Set $x_{i} \equiv X_{t}^{(i)}$ and $y_{\alpha} \equiv f_{t}\left(Y^{(\alpha)}\right)$ and apply the chain rule to get

$$
\begin{aligned}
& \frac{\mathrm{d}\left(Z_{t}^{\varphi} J_{t}^{\varphi}\right)}{J_{t}^{\varphi}}=\left[\sum_{i}\left(\mathrm{~d} M_{t}^{(i)}+F_{t}^{(i)}\right) \partial_{x_{i}}\right. \\
& \left.\quad+\sum_{i} \mathrm{~d} q_{t}^{(i)}\left(\frac{\kappa}{2} \partial_{x_{i}}^{2}+2 \sum_{\alpha}\left[\frac{1}{y_{\alpha}-x_{i}} \partial_{y_{\alpha}}-\frac{\delta_{\alpha}}{\left(y_{\alpha}-x_{i}\right)^{2}}\right]\right)\right] Z_{t}^{\varphi}
\end{aligned}
$$

- First use of the null-vector equation : identification of $\psi$.

Let us concentrate for a moment on the familiar chordal SLE case, for which $n=1$. The drift term $F_{t}^{(1)}$ is known to be zero. The boundary conditions also change at $\infty$ (the endpoint of the interface) and there is an operator there, that we have not written explicitly because the notation is heavy enough. Anyway, $Z_{t}$ is a two-point function with one of the fields at infinity, so it is a constant. For chordal SLE, the drift term in the Ito derivative of the putative martingale vanishes if and only if

$$
\left(\frac{\kappa}{2} \partial_{x}^{2}+2 \sum_{\alpha}\left[\frac{1}{y_{\alpha}-x} \partial_{y_{\alpha}}-\frac{\delta_{\alpha}}{\left(y_{\alpha}-x\right)^{2}}\right]\right) Z_{t}^{\varphi}=0
$$

where for simplicity we have written $x \equiv x_{1}$.

Comparison with eq. (5) implies that $\psi$ has a vanishing descendant at level two and has conformal weight $h=\frac{6-\kappa}{2 \kappa} \equiv h_{1}(\kappa)$ :

$$
\psi(x) \equiv \varphi_{h_{1}(\kappa)}(x) .
$$

The central charge is $c=\frac{(6-\kappa)(3 \kappa-8)}{16 \kappa}$.

This is of course nothing but the correlation function formalism version of the original argument relating SLE to CFT, which was given in the operator formalism, see [7].

- Second use of the null-vector equation.

Now that $\psi$ has been identified, we can return to the general case, with an 
arbitrary number $n$ of growing curves. Each growing curve has its own field $\psi$ and each field $\psi$ comes with its differential equation, which is eq. (15) but for $l+n-1$ spectator fields, the $l$ fields $\varphi$ and the $n-1$ other $\psi$ 's. So $Z_{t}^{\varphi}$ is annihilated by the $n$ differential operators

$$
\frac{\kappa}{2} \partial_{x_{i}}^{2}+2 \sum_{\alpha}\left[\frac{1}{y_{\alpha}-x_{i}} \partial_{y_{\alpha}}-\frac{\delta_{\alpha}}{\left(y_{\alpha}-x_{i}\right)^{2}}\right]+2 \sum_{j \neq i}\left[\frac{1}{x_{j}-x_{i}} \partial_{x_{j}}-\frac{h_{1}(\kappa)}{\left(x_{j}-x_{i}\right)^{2}}\right] .
$$

We can use this to get a simplified formula

$$
\mathrm{d}\left(Z_{t}^{\varphi} J_{t}^{\varphi}\right)=J_{t}^{\varphi} \mathcal{P} Z_{t}^{\varphi}, \quad \mathrm{d} Z_{t}=\mathcal{P} Z_{t}
$$

where $\mathcal{P}$ is the first order differential operator

$$
\sum_{i}\left[\left(\mathrm{~d} M_{t}^{(i)}+F_{t}^{(i)}\right) \partial_{x_{i}}-2 \mathrm{~d} q_{t}^{(i)}\left(\sum_{j \neq i}\left[\frac{1}{x_{j}-x_{i}} \partial_{x_{j}}-\frac{h_{1}(\kappa)}{\left(x_{j}-x_{i}\right)^{2}}\right]\right)\right] .
$$

The formula for $Z_{t}$ is just the special case $l=0$.

- Final application of Ito's formula.

$$
\mathrm{d}\left(\frac{Z_{t}^{\varphi}}{Z_{t}} J_{t}^{\varphi}\right)=J_{t}^{\varphi} \mathcal{Q}\left(\frac{Z_{t}^{\varphi}}{Z_{t}}\right)
$$

where $\mathcal{Q}$ is the first order differential operator

$$
\sum_{i}\left[\mathrm{~d} M_{t}^{(i)}+F_{t}^{(i)}-\kappa \mathrm{d} q_{t}^{(i)}\left(\partial_{x_{i}} \log Z_{t}\right)-2 \sum_{j \neq i} \frac{\mathrm{d} q_{t}^{(j)}}{x_{i}-x_{j}}\right] \partial_{x_{i}}
$$

The martingale property is satisfied if and only if the drift terms vanish.

\subsection{Main claim}

To summarize, we have shown that the system

$$
\mathrm{d} f_{t}(z)=\sum_{i} \frac{2 \mathrm{~d} q_{t}^{(i)}}{f_{t}(z)-X_{t}^{(i)}} \quad, \quad \mathrm{d} X_{t}^{(i)}=\mathrm{d} M_{t}^{(i)}+F_{t}^{(i)}
$$

admits conditioned correlation functions from CFT as martingales if and only if

$$
F_{t}^{(i)}=\kappa \mathrm{d} q_{t}^{(i)}\left(\partial_{x_{i}} \log Z_{t}\right)+2 \sum_{j \neq i} \frac{\mathrm{d} q_{t}^{(j)}}{x_{i}-x_{j}}
$$


where $Z_{t}$ is a partition function. It is under this condition that it describes the growth of $n$ interfaces in a way compatible with statistical mechanics and conformal field theory.

In fact, we have used a special family of correlators. But the same argument applies to all operators (hence the "if" part). Of special interest in the sequel will be the case when $\mathcal{O}$ is a topological observable, for instance taking value 1 if the interface forms a given arch system and 0 otherwise. No Jacobian is involved for such observables and the numerator looks again like a partition function.

\subsection{The moduli space}

From the definition of $Z_{t}$ as a correlation of primary fields with null descendants at level 2 , it is clear that properties $i$ ), $i i$ ), iii) are satisfied, except maybe for the quantization of the possible scaling dimensions of $Z_{t}$, to which we turn now.

This is standard material from CFT and we include it here for completeness.

The correlator $\left\langle\varphi_{h_{\infty}}(\infty) \psi\left(x_{1}\right) \cdots \psi\left(x_{n}\right)\right\rangle$ on the real line satisfies $n$ differential equations. We shall recall why the space of simultaneous solutions which have global conformal invariance has dimension

$$
\left(\begin{array}{c}
n \\
m
\end{array}\right)-\left(\begin{array}{c}
n \\
m-1
\end{array}\right)=(n+1-2 m) \frac{n !}{m !(n-m+1) !}
$$

if $h_{\infty}=h_{n-2 m}(\kappa)$ for some nonnegative integer $m \leq n / 2$ and has dimension 0 otherwise. This will end the derivation of our proposal and match the counting of arches.

At the end of the background on conformal invariance, we mentioned fusion rules: when $\varphi_{h_{1}(\kappa)}(x)$ and a $\varphi_{h_{j}(\kappa)}(y)$ are brought close together, they can be expanded in a basis of local operators that can be grouped in conformal families. We also recalled why the weight $h^{\prime}$ of the primaries in each conformal family had to satisfy $3\left(h_{j}(\kappa)-h^{\prime}\right)^{2}-\left(2 h_{1}(\kappa)+1\right)\left(h_{j}(\kappa)+h^{\prime}\right)=$ $h_{1}(\kappa)\left(h_{1}(\kappa)-1\right)$, so that only two conformal families can appear in a fusion with $\varphi_{h_{1}(\kappa)}$. The two conformal weights are easily found to be $h^{\prime}=h_{j \pm 1}(\kappa)$. Furthermore, $h_{0}(\kappa)=0$ and one can show that the corresponding field has to be the boundary identity operator. By global conformal invariance, the only local operator with a non vanishing one point correlator is the identity and boundary two point functions vanish unless the two local fields have the same conformal weight. This takes care of the counting and selection rules for the $n=0,1$ cases. 
One proceeds by recursion. The points are ordered $x_{1}<x_{2} \cdots<x_{n}$. If $n \geq 2$ then move $x_{2}$ close the $x_{1}$ (for instance by a global conformal transformation) and fuse to get an expansion for local fields at $x_{1}$ say. Only the conformal families of $\varphi_{h_{1 \pm 1}(\kappa)}$ appear. If $n=2$ this fixes the weight of the field at $\infty$. If $n \geq 3$, iterate. This leads immediately to the selection rules mentioned above : the field at infinity has to be a $\varphi_{h_{n-2 m}(\kappa)}$. The dimension is nothing but the number of path of $n$ steps \pm 1 from 0 to $n-2 m$ on the nonnegative integers, a standard combinatorial problem whose answer is $\left(\begin{array}{c}n \\ m\end{array}\right)-\left(\begin{array}{c}n \\ m-1\end{array}\right)$. The efficient way to do the counting is by the reflection principle. The possible outcomes of each fusion can be encoded in a socalled Bratelli diagram:

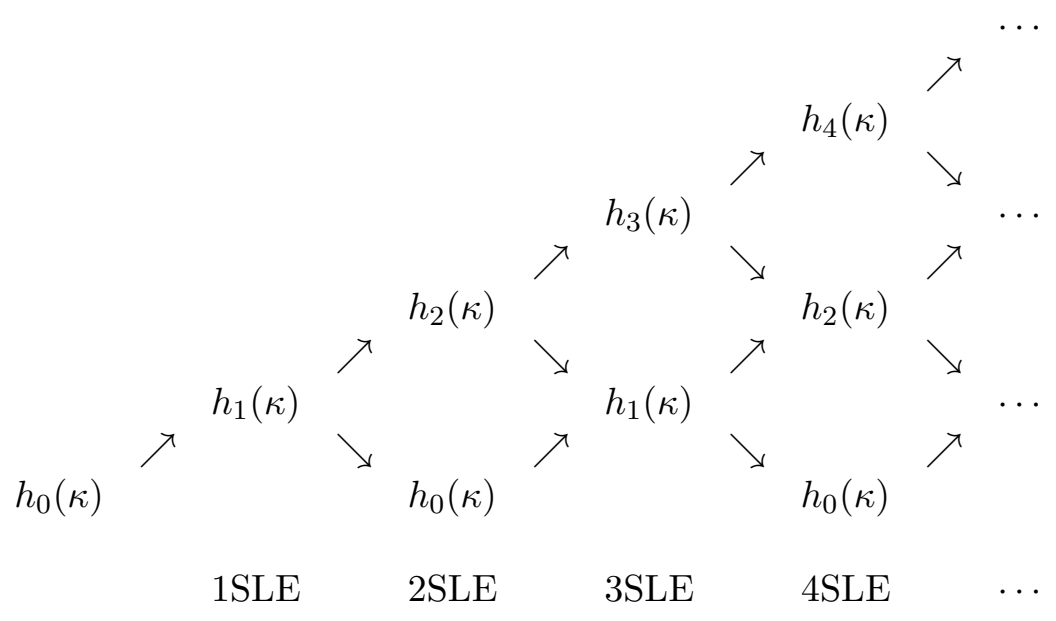

This is totally parallel to the discussion of composition of $n$ spins $1 / 2$ for the representation theory of the Lie algebra of rotations. The multiplicity is exactly one when $m=0$ which corresponds to the partition function (4) and to the insertion of the operator $\varphi_{h_{n}(\kappa)}$ at infinity, toward which the $n$ interfaces run.

What is not proved here is that the different paths lead to a basis of solutions of the $n$ partial differential equations, but it is true. Each path corresponds to a succession of choices of a single conformal family, one at each fusion step. Let us mention in advance that multi SLE processes, i.e. the consideration of multiple interfaces, will lead to the definition of another basis with a topological interpretation. 


\section{Multiple SLEs describing several interfaces}

\subsection{Double SLEs}

The case of double SLEs is instructive and simple to analyze. Although double SLEs is sometimes interesting for its own sake, the purpose of this section is to give easy examples to guide the study of the general case.

\subsubsection{SLEs and Bessel processes}

To specify the process we have to specify the partition function $Z$. There are only two possible choices corresponding to two different type of boundary conditions, or alternatively to two different fields inserted at infinity:

$$
\left\langle h_{\infty}\left|\psi\left(X_{1}\right) \psi\left(X_{2}\right)\right| 0\right\rangle=\text { const. } \times\left(X_{1}-X_{2}\right)^{\Delta}
$$

where the exponent is $\Delta=h_{\infty}-2 h_{1}(\kappa)$ and the constant will be fixed to 1 from now on. According to CFT fusion rules, $h_{\infty}$ can only be either $h_{2}(\kappa)=\frac{8-\kappa}{\kappa}$ or $h_{0}(\kappa)=0$. The exponent becomes $\Delta=2 / \kappa$ or $\Delta=\frac{\kappa-6}{\kappa}$ respectively, so that we have two basic choices for $Z$ :

$$
Z_{0} \equiv\left(X_{1}-X_{2}\right)^{(\kappa-6) / \kappa} \quad \text { or } \quad Z_{2} \equiv\left(X_{1}-X_{2}\right)^{2 / \kappa}
$$

As we shall see, choosing $Z_{0}$ selects configurations with no curve ending at infinity - so that we are actually describing standard chordal SLE joining to the two initial positions of $X_{1}$ and $X_{2}$ - while choosing $Z_{2}$ selects configurations with two curves emerging from the initial positions of $X_{1}$ and $X_{2}$ and ending both at infinity.

Up to normalizing the quadratic variation by $d q_{t}^{(i)}=a_{i} d t$ so that the martingales $M^{(i)}$ are simply $d M_{t}^{(i)}=\sqrt{\kappa a_{i}} d B_{t}^{(i)}$ with $d B_{t}^{(i)}$ two independent normalized Brownian motions, our double SLE equations become :

$$
\begin{aligned}
d f_{t}(z) & =\frac{2 a_{1} \mathrm{~d} t}{f_{t}(z)-X_{t}^{(1)}}+\frac{2 a_{2} \mathrm{~d} t}{f_{t}(z)-X_{t}^{(2)}} \\
d X_{t}^{(1)} & =\sqrt{a_{1} \kappa} d B_{t}^{(1)}+\frac{2 a_{2}+\kappa \Delta a_{1}}{X_{t}^{(1)}-X_{t}^{(2)}} \mathrm{d} t \\
d X_{t}^{(2)} & =\sqrt{a_{2} \kappa} d B_{t}^{(2)}+\frac{2 a_{1}+\kappa \Delta a_{2}}{X_{t}^{(2)}-X_{t}^{(1)}} \mathrm{d} t
\end{aligned}
$$

It describes two curves emerging from points $X_{1}=X_{0}^{(1)}$ and $X_{2}=X_{0}^{(2)}$ at speeds parametrized by $a_{1}$ and $a_{2}$. 

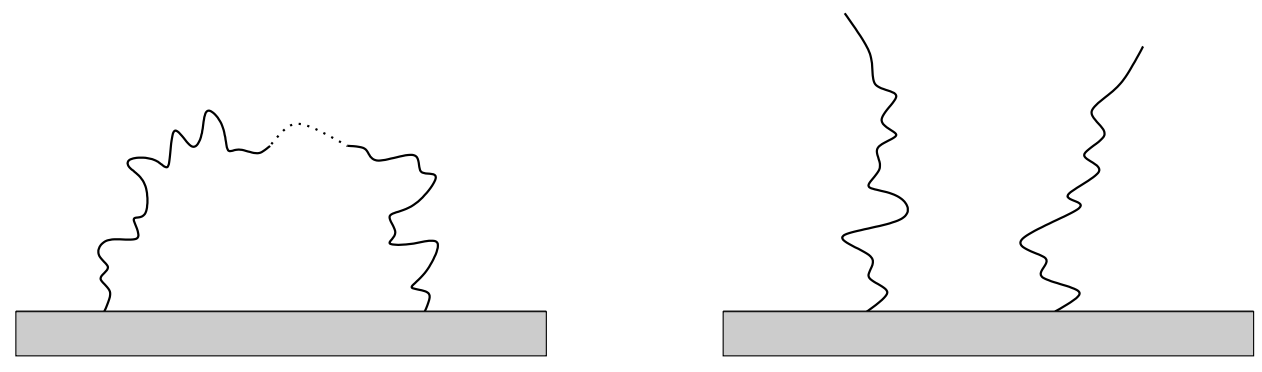

Figure 4: The two geometries for 2SLE: on the left is the case $h_{\infty}=0$ and on the right $h_{\infty}=h_{2}(\kappa)$.

Up to an irrelevant translation, the process is actually driven by the difference $Y_{t}=X_{t}^{(1)}-X_{t}^{(2)}$. Up to a time change, $d s=\kappa\left(a_{1}+a_{2}\right) d t$, this is a Bessel process,

$$
d Y_{s}=\mathrm{d} \tilde{B}_{s}+\frac{\Delta+2 / \kappa}{Y_{s}} \mathrm{~d} s
$$

of effective dimension $d_{\text {eff }}=1+2 \Delta+4 / \kappa$. For $h_{\infty}=h_{2}(\kappa)$ (i.e. $\Delta=2 / \kappa$ ) the dimension is $d_{\mathrm{eff}}=1+8 / \kappa$ and for $h_{\infty}=0$ (i.e. $\Delta=(\kappa-6) / \kappa$ ) it is $d_{\text {eff }}=3-8 / \kappa$. In the physically interesting parameter range $\kappa<8$, the former is $>2$ and the latter is $<2$. Recall now that a Bessel process is recurrent (not recurrent) if its effective dimension is less (greater) than 2. Thus, the driving processes $X_{t}^{(i)}$ hit each other almost surely in the case $h_{\infty}=0$ and they don't hit (a.s.) in the case $h_{\infty}=h_{2}(\kappa)$. Since the hitting of driving processes means hitting of the SLE traces, this teaches us that case $h_{\infty}=0$ describes a single curve joining $X_{1}$ and $X_{2}$ while case $h_{\infty}=h_{2}(\kappa)$ describes two curves converging toward infinity.

Notice that previous results are independent of $a_{1}$ and $a_{2}$, provided their sum does not vanishes. We also observe that setting $a_{1}=1$ and $a_{2}=0$ (or vice versa) one recovers an $\operatorname{SLE}(\kappa ; \kappa \Delta)$. Recall that if $h_{\infty}=0$ then $\rho=\kappa \Delta=\kappa-6$ corresponds to an ordinary chordal SLE from $X_{1}$ to $X_{2}$. Our double SLEs with $h_{\infty}=0$ corresponds to one chordal SLE seen from both ends and the fact that the tips of the traces hit is natural. The other case, $h_{\infty}=h_{2}(\kappa)$ corresponds to $\rho=\kappa \Delta=2$ and since the driving processes can not hit, the process can be defined for all $t \geq 0$. Assuming that $\int_{0}^{\infty}\left(a_{1}+\right.$ $\left.a_{2}\right) \mathrm{d} t=\infty$, the capacity of the hulls grow indefinitely and (at least one of) the SLE traces go to infinity.

The two possible geometries are illustrated in figure 4 . 


\subsubsection{A mixed case for 2SLE}

Because of its simplicity, we use double SLE as a testing ground for mixed correlation functions. So we consider the sum

$$
Z=\lambda Z_{0}+\mu Z_{2}
$$

with both $\lambda$ and $\mu$ positive. As already mentioned, the interpretation of $Z$ as the continuum limit of partition functions of lattice models is unclear since $Z_{0}$ and $Z_{2}$ do not scale the same way. We nevertheless study it to illustrate ways of computing (arch or geometry) probabilities. As one may expect, we no longer have an almost sure global geometry but rather nontrivial probabilities for the two geometries: either no curve at infinity or two curves converging there.

Let $\tau=\inf \left\{t \geq 0: X_{t}^{(1)}=X_{t}^{(2)}\right\}$ be the stopping time which indicates the hitting of the driving processes - and thus of the two curves. We can define the driving processes as solutions of the 2SLE system on the (random) time interval $t \in[0, \tau)$. At the stopping time we define $f_{\tau}(z)=\lim _{s \uparrow \tau} f_{s}(z)$ for such $z \in \mathbb{H}$ that the limit exists and stays in the half plane $\mathbb{H}$. The hull $K_{\tau}$ is defined as the set where the limit doesn't exist or hits $\partial \mathbb{H}$.

The question of geometry is answered by the knowledge of whether the two traces hit, that is whether $\tau<\infty$ or not. Thus we again consider the difference $Y_{t}=X_{t}^{(1)}-X_{t}^{(2)}$, whose Ito derivative is now :

$$
\mathrm{d} Y_{t}=\sqrt{\kappa} \mathrm{d} \tilde{B}_{t}+\frac{2}{Y_{t}}\left(a_{1}+a_{2}\right) \mathrm{d} t+\frac{(\kappa-6) \lambda Y_{t}^{\frac{\kappa-6}{\kappa}}+2 \mu Y_{t}^{\frac{2}{\kappa}}}{Y_{t}\left(\lambda Y_{t}^{\frac{\kappa-6}{\kappa}}+\mu Y_{t}^{\frac{2}{\kappa}}\right)}\left(a_{1}+a_{2}\right) \mathrm{d} t
$$

with $\tilde{B}_{t}=\sqrt{a_{1}} B_{t}^{(1)}-\sqrt{a_{2}} B_{t}^{(2)}$ is a Brownian motion, so that after a time change, $\mathrm{d} s=\left(a_{1}+a_{2}\right) \mathrm{d} t$, the result doesn't depend on $a_{1}$ or $a_{2}$. The last drift term comes from the derivative of $\log Z$.

One might for example try to find the distribution of $\tau$ by its Laplace transform $\mathbb{E}_{Y_{0}=y}\left[e^{-\beta \tau}\right]=f_{\beta}(y)$. By Markov property,

$$
\mathbb{E}\left[e^{-\beta \tau} \mid \mathcal{F}_{t}\right]=e^{-\beta t} f_{\beta}\left(Y_{t}\right)
$$

is a closed martingale on $t \in[0, \tau)$ so requiring its Ito drift to vanish leads to the differential equation

$$
\left(-\frac{\beta}{a_{1}+a_{2}}+\left(\frac{2}{y}+\frac{(\kappa-6) \lambda+2 \mu y^{(8-\kappa) / \kappa}}{\lambda+\mu y^{(8-\kappa) / \kappa}}\right) \partial_{y}+\frac{\kappa}{2} \partial_{y}^{2}\right) f_{\beta}(y)=0
$$


The result depends only on $\beta /\left(a_{1}+a_{2}\right)$. We conclude that the distribution of $\left(a_{1}+a_{2}\right) \tau$, the capacity of the final hull $K_{\tau}$, is independent of the speeds of growth $a_{1}$ and $a_{2}$. Also the result depends on $\lambda$ and $\mu$ only through $\mu / \lambda$.

In particular we want to take $\beta \downarrow 0$ to compute the probability that the traces hit. Constant functions solve the differential equation but another linearly independent solution has the correct boundary values $f_{0}(0)=1$ and $f_{0}(\infty)=0$, namely

$$
\mathbb{P}_{Y_{0}=y}[\tau<\infty]=\lim _{\beta \downarrow 0} \mathbb{E}_{Y_{0}=y}\left[e^{-\beta \tau}\right]=\frac{\lambda}{\lambda+\mu y^{(8-\kappa) / \kappa}}
$$

As expected on general ground, this is the fraction of the two partition functions $\lambda Z_{0}$ and $Z=\lambda Z_{0}+\mu Z_{2}$.

\subsection{Triple and/or quadruple SLEs}

We will give a few more of examples of multiple SLEs. Certain triple and quadruple SLEs are the scaling limits of interfaces in percolation and Ising model with rather natural boundary conditions. These models will be considered in section 8.3. Here we study triple and quadruple SLEs for their own sake. We restrict ourselves to $\kappa<8$.

\subsubsection{SLE (pure) configurations}

Partition functions with $n=3$ have only two possible scaling behaviors depending whether the weight $h_{\infty}$ of the field at infinity equals either to $h_{3}(\kappa)=\frac{3(10-\kappa)}{2 \kappa}$ or to $h_{1}(\kappa)=\frac{6-\kappa}{2 \kappa}$. This follows from CFT fusion rules. For reasons already explained we shall not mixed them.

The case $h_{\infty}=h_{3}(\kappa)$ is the simplest. There is only one possible partition function with this scaling, namely

$$
\left[\left(X_{2}-X_{1}\right)\left(X_{3}-X_{1}\right)\left(X_{3}-X_{2}\right)\right]^{2 / \kappa}
$$

It is expected to correspond to configurations with three curves starting at initial positions $X_{1}, X_{2}$ and $X_{3}$ and converging toward infinity.

The case $h_{\infty}=h_{1}(\kappa)$ is more interesting since the space of such partition functions is of dimension two and coincides with the space of conformal block with 4 insertions of boundary operators $\psi$, with one localized at $X_{4}=\infty$ :

$$
\left\langle\psi\left(X_{4}\right) \psi\left(X_{3}\right) \psi\left(X_{2}\right) \psi\left(X_{1}\right)\right\rangle
$$

We assume the points to be ordered $X_{1}<X_{2}<X_{3}<X_{4}$. The associated process should describe a family of two curves joining any pair of adjacent 

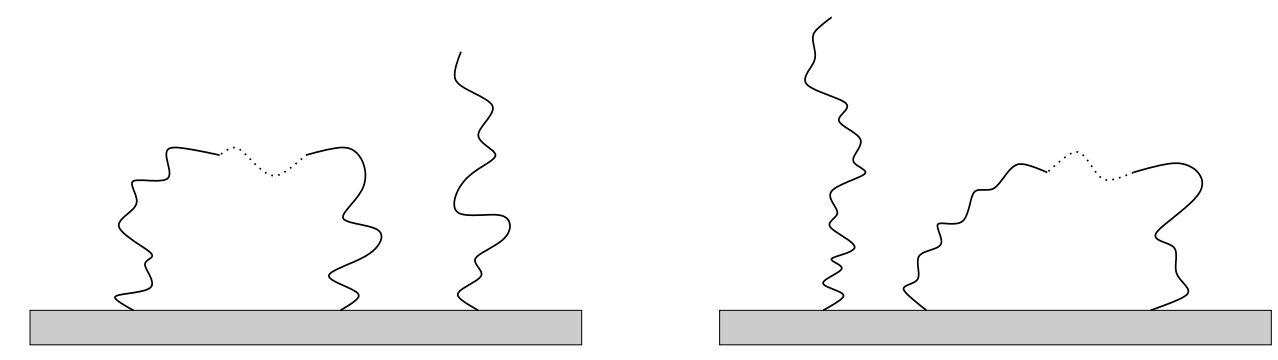

Figure 5: For $h_{\infty}=h_{1}(\kappa)$ the curves of 3SLE join either $\left[X_{1} X_{2}\right]$ and $\left[X_{3} X_{4}\right]$ (on the left) or $\left[X_{4} X_{1}\right]$ and $\left[X_{2} X_{3}\right]$ (on the right).

points without crossing. There are thus two possible topologically distinct geometries: either the curves join the pairs $\left[X_{1} X_{2}\right]$ and $\left[X_{3} X_{4}\right]$ or they join $\left[X_{4} X_{1}\right]$ and $\left[X_{2} X_{3}\right]$, see figure 5. As expected, the number of topologically distinct configuration equals that of conformal blocks, namely two. Notice that the last process is the same as a $4 \mathrm{SLE}$ but with the speed $a_{4}$ vanishing, see figure 6.

By conformal invariance we may normalize the points so that $X_{1}=0$, $X_{2}=x, X_{3}=1$ and $X_{4}=\infty$ with $0<x<1$. We have two distinct topological configurations and we thus have to identify the two corresponding pure partition functions. This is will be done by specifying the way the partition functions behave when points are fused together. By construction these partition functions may be written as correlation functions

$$
Z(x)=\langle\psi(\infty) \psi(1) \psi(x) \psi(0)\rangle
$$

so that their behavior when points are fused are governed by CFT fusion rules. As a consequence, $Z(x)$ behave either as $x^{\frac{\kappa-6}{\kappa}}$ or as $x^{\frac{2}{\kappa}}$ as $x \rightarrow 0$.
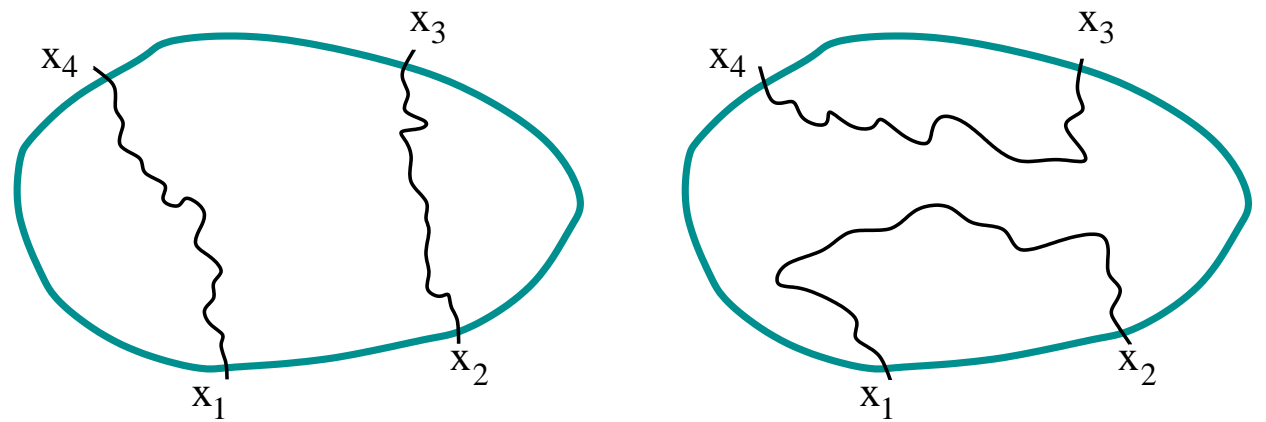

Figure 6: Arch configurations for four SLE processes in an arbitrary domain. 
We select the pure partition functions $Z_{I}$ and $Z_{I I}$ by demanding that:

$$
\begin{aligned}
Z_{I}(x) & =x^{\frac{\kappa-6}{\kappa}} \times[1+\cdots], & & \text { as } x \rightarrow 0 \\
& =(1-x)^{\frac{2}{\kappa}} \times[\text { const. }+\cdots], & & \text { as } x \rightarrow 1
\end{aligned}
$$

and $Z_{I I}(x)=Z_{I}(1-x)$ so that

$$
\begin{aligned}
Z_{I I}(x) & =x^{\frac{2}{\kappa}} \times[\text { const. }+\cdots], & & \text { as } x \rightarrow 0 \\
& =(1-x)^{\frac{\kappa-6}{\kappa}} \times[1+\cdots], & & \text { as } x \rightarrow 1
\end{aligned}
$$

$Z_{I}$ will turn out to be the pure partition function for configurations in which

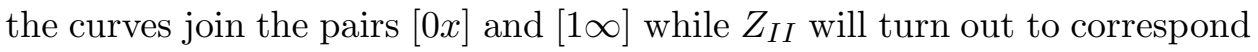
to the configurations $[x 1]$ and $[\infty 0]$. The rationale behind these conditions consists in imposing that the pure partition function possesses the leading singularity, with exponent $(6-\kappa) / \kappa$, when $x$ is approaching the point allowed by the configuration but has subleading singularity, with exponent $2 / \kappa$, when $x$ is approaching the point forbidden by the configuration.

This set of conditions uniquely determines the functions $Z_{I}$ and $Z_{I I}$. These follows from CFT rules but may also be checked by explicitly solving the differential equation that these functions satisfy. Writing $Z(x)=$ $x^{2 / \kappa}(1-x)^{2 / \kappa} G(x)$ yields,

$$
\kappa^{2} x(1-x) G^{\prime \prime}(x)+8 \kappa(1-2 x) G^{\prime}(x)-4(12-\kappa) G(x)=0
$$

so that $G(x)$ is an hypergeometric function and

$$
Z_{I I}(x)=\text { const. } x^{2 / \kappa}(1-x)^{2 / \kappa} F\left(\frac{4}{\kappa}, \frac{12-\kappa}{\kappa} ; \frac{8}{\kappa} \mid x\right)
$$

with the constant chosen to normalize $Z_{I}$ as above. Using this explicit formula one may verify that $Z_{I}(x)$ is effectively a positive number for any $x \in[0 ; 1]$ so it has all expected properties to be a pure partition function.

For $\kappa=4, Z_{I}(x)=\sqrt{(1-x) / x}$ and for $\kappa=2, Z_{I}(x)=\left(1-x^{2}\right) / x^{2}$.

\subsubsection{Arch probabilities}

Let us now compute the probabilities for having one of the two topologically distinct configurations: either $(I)$ with curves joining either $[0 x]$ and $[1 \infty]$ or $(I I)$ with curves joining $[x 1]$ and $[\infty 0]$ as we just discussed. We shall proceed blindly, but the reader should beware that there are subtleties involved. What is computed is the probability for certain $X_{t}^{(i)}$ 's to hit each other. 
What happens at the level of hulls and how the process should be properly continued is not investigated, but is expected to yield the announced probability for arch configuration.

We consider a generic partition function $Z$ which is the sum of the pure partition functions $Z_{I}$ and $Z_{I I}$ :

$$
Z(x)=p_{I} Z_{I}(x)+p_{I I} Z_{I I}(x)
$$

with $p_{I}$ and $p_{I I}$ positive. To specify the $3 \mathrm{SLE}$ (or $4 \mathrm{SLE}$ ) process we need the partition function $Z\left(X_{1}, X_{2}, X_{3}, X_{4}\right)$ which is recover from $Z(x)$ by conformal transformation :

$$
Z\left(X_{1}, X_{2}, X_{3}, X_{4}\right)=\left[\left(X_{4}-X_{2}\right)\left(X_{3}-X_{1}\right)\right]^{\frac{\kappa-6}{\kappa}} Z(X)
$$

with $X$ the harmonic ratio of the four points $X_{1}, X_{2}, X_{3}$ and $X_{4}$ :

$$
X=\left(\frac{X_{1}-X_{2}}{X_{1}-X_{3}}\right)\left(\frac{X_{4}-X_{3}}{X_{4}-X_{2}}\right) \text {. }
$$

Let $M_{I}(x)$ and $M_{I I}(x)=1-M_{I}(x)$ be defined by

$$
M_{I}(x) \equiv p_{I} Z_{I}(x) / Z(x) \quad, \quad M_{I I}(x) \equiv p_{I I} Z_{I I}(x) / Z(x)
$$

By construction the processes $t \rightarrow M_{I}\left(X_{t}\right)$ and $t \rightarrow M_{I I}\left(X_{t}\right)$, with $X_{t}$ the harmonic ratio of the four moving points, are local martingales. Since both $Z_{I}$ and $Z_{I I}$ are positive, $M_{I}$ are $M_{I I}$ are bounded local martingales and thus are martingales.

Let $\tau$ be the stopping time given by the first instant at which a pair of points $X_{t}^{(i)}$ coincide. Then, in configuration $(I)$ we have $\lim _{t} \nearrow_{\tau} X_{t}=0$ while $\lim _{t \nearrow_{\tau}} X_{t}=1$ in configuration $(I I)$. Since, for $\kappa<8, M_{I}(x)$ is such that $\lim _{x \rightarrow 0} M_{I}(x)=1$ but $\lim _{x \rightarrow 1} M_{I}(x)=0$, we obtain that $M_{I}$ evaluated at the stopping time $\tau$ is the characteristic function for events with the topological configuration $(I)$, ie:

$$
\begin{aligned}
\lim _{t \nearrow \tau} M_{I}\left(X_{t}\right) & =\mathbf{1}_{\text {config. }(I)} \\
\lim _{t \nearrow \tau} M_{I I}\left(X_{t}\right) & =\mathbf{1}_{\text {config. }(I I)}
\end{aligned}
$$

Since $M_{I}$ and $M_{I I}$ are martingales, we get the probability of occurrence of configurations of topological type $(I)$ :

$$
\mathbb{P}[\text { config. }(I)]=M_{I}\left(X_{t=0}\right)=\frac{p_{I} Z_{I}(x)}{p_{I} Z_{I}(x)+p_{I I} Z_{I I}(x)}
$$

and similarly for the probabilities of having configuration $(I I)$. As expected they are ratios of partition functions. 


\subsection{Applications to percolation and Ising model}

We are now ready to give an application of triple (or quadruple) SLE to percolation and Ising model. Exploration processes in critical percolation are described by SLEs with $\kappa=6$, as proved in [19]. Interfaces of spin clusters in critical Ising model is believed to correspond to $\kappa=3$ while interfaces of Fortuin-Kasteleyn clusters - which occur in a high temperature expansion of the Ising partition function - are expected to correspond to the dual value $\kappa=16 / 3$.

What we have in mind are these statistical models, defined on the upper half plane, with boundary condition changing operators at the four points $0, x, 1$ and $\infty$. They change the boundary condition from open to closed (or vice versa) in percolation $(\kappa=6)$ and from plus to minus (or vice versa) for Ising model $(\kappa=3)$.

To apply previous results on 4SLE processes to these situations, we have to specify the partition functions $Z(x)$, or equivalently, we have to specify the value of $p_{I}$ and $p_{I I}$. This is done by noticing that these models are left-right symmetric so that for $x=1 / 2$ there is equal probability to find configuration $(I)$ or $(I I)$. Since $Z_{I}(1 / 2)=Z_{I I}(1 / 2)$, we have $p_{I}=p_{I I}=1$, so that the total partition function is $Z(x)=Z_{I}(x)+Z_{I I}(x)$ and the probability of occurrence of configuration $(I)$ for any $0<x<1$ is now:

$$
\mathbb{P}[\text { config. }(I)]=\frac{Z_{I}(x)}{Z_{I}(x)+Z_{I I}(x)} \quad, \quad Z_{I I}(x)=Z_{I}(1-x)
$$

- Percolation corresponds to $\kappa=6$. The boundary changing operator $\psi$ has dimension 0 . The pure partition function $Z_{I}$ has a simple integral representation:

$$
Z_{I}(x)_{\text {perco }}=\frac{\Gamma(2 / 3)}{\Gamma(1 / 3)^{2}} \int_{x}^{1} \mathrm{~d} s s^{-2 / 3}(1-s)^{-2 / 3} .
$$

By construction $Z_{I I}(x)=Z_{I}(1-x)$ also possesses a simple integral representation but, most importantly, it is such that the total partition function is constant, $Z(x)=Z_{I}(x)+Z_{I I}(x)=1$, as expected for percolation. As a consequence we find:

$$
\mathbb{P}[\text { config. }(I)]_{\text {perco }}=\frac{\Gamma(2 / 3)}{\Gamma(1 / 3)^{2}} \int_{x}^{1} \mathrm{~d} s s^{-2 / 3}(1-s)^{-2 / 3}
$$

This is nothing but Cardy percolation crossing formula. 
- Ising spin clusters correspond to $\kappa=3$. The boundary changing operator $\psi$ has dimension $1 / 2$ and may thus be identified with a fermion on the boundary. However the pure partition functions do not correspond to the free fermion conformal block. By solving the differential equation with the appropriate boundary condition we get:

$$
Z_{I}(x)_{\text {spin Ising }}=\text { const } \frac{1-x+x^{2}}{x(1-x)} \int_{x}^{1} \mathrm{~d} y \frac{(y(1-y))^{2 / 3}}{\left(1-y+y^{2}\right)^{2}}
$$

The total partition function $Z_{I}(x)+Z_{I}(1-x)$ is proportional to $\frac{1-x+x^{2}}{x(1-x)}$, which is the free fermion result.

Hence, the Ising configuration probabilities are :

$$
\mathbb{P}[\text { config. }(I)]_{\text {spin Ising }}=\int_{x}^{1} \mathrm{~d} y \frac{(y(1-y))^{2 / 3}}{\left(1-y+y^{2}\right)^{2}} / \int_{0}^{1} \mathrm{~d} y \frac{(y(1-y))^{2 / 3}}{\left(1-y+y^{2}\right)^{2}}
$$

This is nothing but a new - and previously unknown - Ising crossing formula.

- FK Ising clusters correspond to $\kappa=16 / 3$. The operator $\psi$ has then dimension $1 / 16$. The pure partition function are given by:

$$
Z_{I}(x)_{\mathrm{FK} \text { Ising }}=\frac{(1-x)^{3 / 8}}{x^{1 / 8}(1+\sqrt{x})^{1 / 2}}
$$

and the crossing probabilities by:

$$
\mathbb{P}[\text { config. }(I)]_{\text {FK Ising }}=\frac{\sqrt{(1-x)+(1-x)^{3 / 2}}}{\sqrt{x+x^{3 / 2}}+\sqrt{(1-x)+(1-x)^{3 / 2}}}
$$

The other critical random cluster (or Potts) models with $0 \leq Q \leq 4$ have $Q=4 \cos ^{2}\left(\frac{4 \pi}{\kappa}\right), 4 \leq \kappa \leq 8$ and it is straightforward to obtain explicit crossing formulas involving only hypergeometric functions.

\section{4 nSLEs and beyond}

We now comment on how to compute multiple arch probabilities for general nSLEs. This section only aims at giving some hints on how to generalize previous computations. So it shall be sketchy. It is clear that the key point is to identify the pure partition functions - once this is done the rest is routine. As exemplified above by eq.(9) this is linked to CFT fusions. The rules there were that, for a given arch system, fusing two points linked by an arch produces the dominant singularity which means that the two boundary 

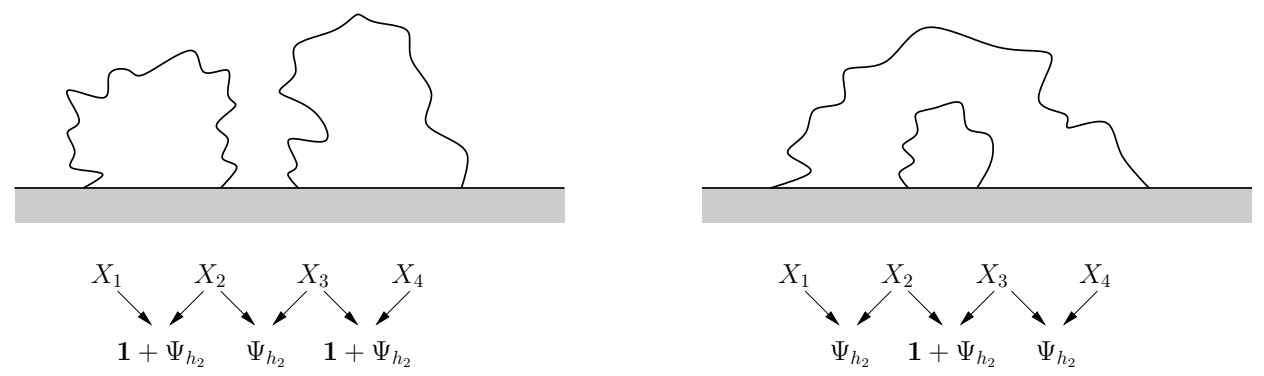

Figure 7: Illustration of the fusion rules corresponding to arch configurations.

operators are fused on the identity operator, whereas fusing two points not linked by an arch produces the subleading singularity which means the fusion of the two boundary fields on the identity should vanish. In general there could be a whole hierarchy of arches with arches in the interior of others, i.e. with a family of self-surrounding arches, the next encircling the previous. So we are lead to propose the following rules.

For a given arch configuration:

- The most interior pair of adjacent pair of points, say $X_{i}, X_{i+1}$ in a family of self-surrounding arches fused into the identity operator, so that the pure partition function evaluated at $X_{i} \simeq X_{i+1}$ should be proportional to $\left(X_{i+1}-\right.$ $\left.X_{i}\right)^{\frac{\kappa-6}{\kappa}}$ times the pure partition function associated to the arch system with the interior arch $\left[X_{i} X_{i+1}\right]$ removed. Symbolically :

$Z_{\text {pure }}\left(\cdots, X_{i} \simeq X_{i+1}, \cdots\right) \simeq$ const. $\left(X_{i+1}-X_{i}\right)^{\frac{\kappa-6}{\kappa}} \times Z_{\text {pure } \backslash\left[X_{i} X_{i+1}\right]}(\cdots, \cdots)$

for $X_{i}$ and $X_{i+1}$ linked by an arch.

- The fusion on the identity of any pair of adjacent points not linked by an arch should vanish, so that the fusion of this pair of points produces the subleading singularity. Symbolically :

$$
Z_{\text {pure }}\left(\cdots, X_{i} \simeq X_{i+1}, \cdots\right) \simeq \text { const. }\left(X_{i+1}-X_{i}\right)^{\frac{2}{\kappa}}+\cdots
$$

for $X_{i}$ and $X_{i+1}$ for not linked by an arch.

We do not have a complete proof that these rules fully determine the pure partition functions but we checked it on a few cases, see figure $\mathbf{7}$

Here are a few samples. We shall give the relation between the pure partition and the CFT conformal blocks indexed by the corresponding Bratelli diagram. For $n=4$, we may have the following arch systems $\left[X_{1} X_{2}\right]\left[X_{3} X_{4}\right]$ or 
$\left[X_{1}\left[X_{2} X_{3}\right] X_{4}\right]$. (A given geometrical configuration may correspond to different arch systems depending at which location we open the closed boundary. But they are all equivalent to these two up to an order preserving relabeling of the points. For instance $\left[X_{4} X_{1}\right]\left[X_{2} X_{3}\right]$ is equivalent to $\left[X_{1}\left[X_{2} X_{3}\right] X_{4}\right]$.) Applying the previous rules we get:

$$
\begin{aligned}
Z_{\left[X_{1}\left[X_{2} X_{3}\right] X_{4}\right]} & =\left\langle{ }_{\left[h_{0}\right]} \psi\left(X_{1}\right)_{\left[h_{1}\right]} \psi\left(X_{2}\right)_{\left[h_{2}\right]} \psi\left(X_{3}\right)_{\left[h_{1}\right]} \psi\left(X_{4}\right)_{\left[h_{0}\right]}\right\rangle \\
Z_{\left[X_{1} X_{2}\right]\left[X_{3} X_{4}\right]} & =\left\langle{ }_{\left[h_{0}\right]} \psi\left(X_{1}\right)_{\left[h_{1}\right]} \psi\left(X_{2}\right)_{\left[h_{0}\right]} \psi\left(X_{3}\right)_{\left[h_{1}\right]} \psi\left(X_{4}\right)_{\left[h_{0}\right]}\right\rangle \\
& +\omega\left\langle_{\left[h_{0}\right]} \psi\left(X_{1}\right)_{\left[h_{1}\right]} \psi\left(X_{2}\right)_{\left[h_{2}\right]} \psi\left(X_{3}\right)_{\left[h_{1}\right]} \psi\left(X_{4}\right)_{\left[h_{0}\right]}\right\rangle
\end{aligned}
$$

where the indices $h_{m}, m=0,1, \cdots$ refer to the corresponding points in the Bratelli diagram, i.e. to the weights $h_{m}(\kappa)$ of the intermediate Virasoro modules. The coefficient $\omega$ is fully determined, in terms of CFT fusion coefficients, by demanding that the fusion of $X_{2}$ and $X_{3}$ on the identity vanishes.

One may go on and solve for the pure partition functions in few other cases. A particularly simple example with $n=6$ is given by :

$$
\begin{gathered}
Z_{\left[X_{1}\left[X_{2}\left[X_{3} X_{4}\right] X_{5}\right] X_{6}\right]}= \\
\left\langle{ }_{\left[h_{0}\right]} \psi\left(X_{1}\right)_{\left[h_{1}\right]} \psi\left(X_{2}\right)_{\left[h_{2}\right]} \psi\left(X_{3}\right)_{\left[h_{3}\right]} \psi\left(X_{4}\right)_{\left[h_{2}\right]} \psi\left(X_{5}\right)_{\left[h_{1}\right]} \psi\left(X_{6}\right)_{\left[h_{0}\right]}\right\rangle
\end{gathered}
$$

As can be seen on these examples, there is no simple relation between arch systems and Bratelli diagrams and the change of basis for one to the other is quite involved. The only simple rule we find is that the pure partition function for a unique family of self-surrounding arches is a pure conformal block corresponding to a unique Bratelli diagram.

\section{References}

[1] O. Schramm, Israel J. Math., 118, 221-288, (2000);

[2] S. Rohde, O. Schramm "Basic Properties of SLE" Ann. Math., to appear, 2001 arXiv:math.PR/0106036.

[3] W. Werner "Random planar curves and Schramm-Loewner evolutions" In Lectures on probability theory and statistics, vol. 1840 of Lecture Notes in Math., p. 107-195. arXiv:math.PR/0303354

Springer, Berlin, 2004 .

[4] G. Lawler, O. Schramm, W. Werner "Conformal restriction. The chordal case" to appear in JAMS arXiv:math.PR/0209343. 
[5] G. Lawler, O. Schramm and W. Werner, (I): Acta Mathematica 187 (2001) 237-273; arXiv:math.PR/9911084

G. Lawler, O. Schramm and W. Werner, (II): Acta Mathematica 187 (2001) 275-308; arXiv:math.PR/0003156

G. Lawler, O. Schramm and W. Werner, (III): Ann. Henri Poincaré 38 (2002) 109-123. arXiv:math.PR/0005294.

[6] J. Dubedat "SLE $(\kappa, \rho)$ martingales and duality" to appear in Ann. Probab., 2003 [arXiv:math.PR/].

[7] M. Bauer and D. Bernard, "Conformal field theories of stochastic Loewner evolutions" Commun. Math. Phys. 239 (2003) 493 arXiv:hep-th/0210015.

[8] M. Bauer and D. Bernard, "SLE(kappa) growth processes and conformal field theories" Phys. Lett. B $\mathbf{5 4 3}$ (2002) 135 arXiv:math-ph/0206028.

[9] R. Friedrich and W. Werner, "Conformal restriction, highest weight representations and SLE", arXiv:math-ph/0301018.

[10] M. Bauer and D. Bernard, "SLE martingales and the Virasoro algebra," Phys. Lett. B 557 (2003) 309 arXiv:hep-th/0301064.

[11] M. Bauer and D. Bernard, "Conformal transformations and the SLE partition function martingale" Annales Henri Poincare 5 (2004) 289 arXiv:math-ph/0305061.

[12] M. Bauer and D. Bernard, "CFTs of SLEs: The radial case" Phys. Lett. B 583 (2004) 324 arXiv:math-ph/0310032.

[13] M. Bauer and D. Bernard, "SLE, CFT and zig-zag probabilities" arXiv:math-ph/0401019.

[14] M. Bauer, D. Bernard and J. Houdayer, "Dipolar SLEs" arXiv:math-ph/0411038.

[15] J. Cardy, "Stochastic Loewner evolution and Dysons circular ensembles", J. Phys. A: Math. Gen. 36 (2003) L379-L386 arXiv:math-ph/0301039.

[16] J. Dubedat "Some remarks on commutation relations for SLE" (2004) arXiv:math.PR/0411299. 
[17] A. Belavin, A. Polyakov, A. Zamolodchikov: "Infinite conformal symmetry in two-dimensional quantum field theory", Nucl. Phys. B241, 333-380, (1984).

[18] P. Di Francesco, P. Mathieu, D. Sénéchal: "Conformal Field Theory" Springer-Verlag New York, Inc., 1997

[19] S. Smirnov: "Critical percolation in the plane" C. R. Acad. Sci. Paris Sér. I Math., 333 (2001), no. 3, 239-244. 